ALEA, Lat. Am. J. Probab. Math. Stat. 16, 361-388 (2019)

DOI: $10.30757 /$ ALEA.v16-13

\title{
Boundary driven Brownian gas
}

\section{Lorenzo Bertini and Gustavo Posta}

\author{
Università degli Studi di Roma "La Sapienza" \\ P.le A. Moro 5, \\ 00185 Roma, Italy. \\ E-mail address: bertini@mat.uniroma1.it \\ URL: http://www.mat. uniroma1.it/people/bertini/ama/ \\ Università degli Studi di Roma "La Sapienza" \\ P.le A. Moro 5, \\ 00185 Roma, Italy. \\ E-mail address: gustavo.posta@uniroma1.it \\ URL: http://www.mat.uniroma1.it/ posta
}

\begin{abstract}
We consider a gas of independent Brownian particles on a bounded interval in contact with two particle reservoirs at the endpoints. Due to the Brownian nature of the particles, infinitely many particles enter and leave the system in each time interval. Nonetheless, the dynamics can be constructed as a Markov process with continuous paths on a suitable state space. If $\lambda_{0}$ and $\lambda_{1}$ are the chemical potentials of the boundary reservoirs, the stationary distribution (reversible if and only if $\lambda_{0}=\lambda_{1}$ ) is a Poisson point process with intensity given by the linear interpolation between $\lambda_{0}$ and $\lambda_{1}$. We then analyze the empirical flow that it is defined by counting, in a time interval $[0, t]$, the net number of particles crossing a given point $x$. In the stationary regime we identify its statistics and show that it is given, apart an $x$ dependent correction that is bounded for large $t$, by the difference of two independent Poisson processes with parameters $\lambda_{0}$ and $\lambda_{1}$.
\end{abstract}

\section{Introduction}

Stationary non-equilibrium states, that describe a steady flow thought some system, are the simplest examples of non-equilibrium phenomena. The prototypical example is the case of an iron rod whose endpoints are thermostated at two different temperatures. For these systems the paradigm of statistical mechanics, i.e. the Boltzman-Gibbs formula, is not applicable and an analysis of the dynamics is

Received by the editors June 5th, 2017; accepted February 24 th, 2019.

2010 Mathematics Subject Classification. 60K35, 60J70, 82B21.

Key words and phrases. Continuum particles systems, Poisson point processes, Currents.

This work has been supported by the PRIN 20155PAWZB "Large Scale Random Structures". 
required to construct the relevant statical ensambles. In the last years, considerable progress on stationary non-equilibrium states has been achieved by considering as basic model stochastic lattice gases, that consist on a collection of interacting random walks on the lattice, while the reservoirs are modeled by birth and death processes on the boundary sites. The analysis of such boundary driven models has revealed a few different features with respect to the their equilibrium, i.e. reversible, counterpart such as the presence of long range correlations in the stationary measure even at high temperature and the occurrence of dynamical phase transitions that can be spotted by analyzing the large deviation properties of the empirical current. We refer to Bertini et al. (2015) and to Mallick (2015) for reviews on these topics.

The present purpose is the construction of boundary driven models for particles living on the continuum and not on the lattice, the main issue being the modeling of the boundary reservoirs. If we consider Brownian motion as basic reference for the bulk dynamics, the boundary reservoirs need to inject Brownian particles on the system and accordingly each particle entering the system immediately leaves it. Nonetheless, it should be possible to define, possibly through a limiting procedure, a suitable model of the reservoirs in which, out of the infinitely many entrances on a given time interval, the number of particles that managed to move away from the boundary at least by $\varepsilon>0$ is finite with probability one.

We here pursue the above program in the simple case of independent particles in the interval $(0,1)$, the resulting process is referred to as the boundary driven Brownian gas. For interacting Brownian motions, the model of the boundary reservoirs should be analogous to the one here introduced. We present several alternative constructions of the boundary driven Brownian gas. We define directly the dynamics as a Markov process by specifying explicitly its transition function, we provide a construction from the excursion process of a single Brownian motion on $(0,1)$, and give a graphical construction of its restriction to the sub-intervals $[a, 1-a]$, $0<a<1 / 2$ with a Poissonian law of the entrance times. We finally obtain this process by considering the limit of $n$ independent sticky Brownian motions on $[0,1]$ with stickiness parameter of order $1 / n$. From the last convergence we deduce in particular the continuity of the paths of the boundary driven Brownian gas.

Since there is no interaction among the particles, the invariant measure of the boundary driven Brownian gas is simply a Poisson point process on $(0,1)$. More precisely, letting $\lambda_{0}$ and $\lambda_{1}$ being the chemical potentials of the boundary reservoirs, the stationary distribution (reversible if and only if $\lambda_{0}=\lambda_{1}$ ) is a Poisson point process with intensity given by the linear interpolation between $\lambda_{0}$ and $\lambda_{1}$.

For stochastic lattice gases, a relevant dynamical observable is the empirical flow that is defined by counting, in a time interval $[0, t]$, the net number of particles crossing a given bond. In order to define the empirical flow for the boundary driven Brownian gas, given $x \in(0,1)$ and $\varepsilon>0$ we first count the total net number of crossing of the interval $(x-\varepsilon, x+\varepsilon)$ in the time interval $[0, t]$. The empirical flow at $x$ is then defined by taking the limit $\varepsilon \rightarrow 0$. In the stationary regime we identify its statistics and show that it is given, apart an $x$ dependent correction that is bounded for large $t$, by the difference of two independent Poisson processes with parameters $\lambda_{0}$ and $\lambda_{1}$. A similar result in the context of stochastic lattice gases is proven in Ferrari and Fontes (1994). 
The construction boundary driven Brownian gas presents some technical issues. In order to have a well defined dynamics with finitely many particles on each compact subset of $(0,1)$, the set of allowed configurations needs to be properly chosen. The natural topology on such state space is not Polish and additional analysis is required. Moreover, the boundary driven Brownian gas is not a Feller process and the regularity of its paths has to be properly investigated. As outlined above, we deduce the continuity of the paths by considering the limit of independent sticky Brownian motions, however the lack of the Feller property has to be circumvented in order to identify the finite dimensional distributions.

Although not here discussed the boundary driven Brownian gas could be obtained by considering the Poisson limit of boundary driven lattice gases. Consider for instance the symmetric zero range process on $\{0, \ldots, N\}$ where particles are created at the site 0 (resp. $N$ ) with rate $\lambda_{0} / N$ (resp. $\left.\lambda_{1} / N\right)$. By considering a sequence of initial configurations with bounded number of particles and performing the diffusive rescaling of space/time, it should be possible to show that in the limit $N \rightarrow+\infty$ the process converges to the boundary driven Brownian gas.

The dynamics of infinitely many stochastic particle has a long and rich history, dating back to Dobrušin (1956). The ergodic properties of independent particles have been early discussed in Shiga and Takahashi (1973/74). More recently, an approach based on Dirichlet forms has been introduced in Albeverio et al. (1998) and successively extensively developed. We also mention Hiraba (1996), where a model with immigration is considered. The case of Brownian hard spheres is analyzed in Fradon et al. (2000) and in Tanemura (1996).

\section{Construction and basic properties}

A configuration of the system is given by specifying the positions of all the particles. Regarding these particles as indistinguishable, we identify a configuration with the integer valued measure on $(0,1)$ obtained by giving unit mass at the position of each particle. To construct the dynamics, we need to specify a suitable temperedness condition on the set of allowed configurations.

We denote by $C_{K}(0,1)$ the set of continuous function on $(0,1)$ with compact support. As usual, $C_{0}(0,1)$ is the closure in the uniform norm of $C_{K}(0,1)$. We identify $C_{0}(0,1)$ with the functions in $C[0,1]$ that vanish at the endpoints. Letting $m \in C_{0}(0,1)$ be the function $m(x):=x(1-x)$, we define $\Omega$ as the set of $\mathbf{Z}_{+} \cup\{+\infty\}$ valued (Radon) measures $\omega$ on $(0,1)$ satisfying $\omega(m)<+\infty$. In particular, an element $\omega \in \Omega$ can be written as $\omega=\sum_{k} \delta_{x_{k}}$ for some $x_{1}, x_{2}, \cdots \in(0,1)$ such that $\sum_{k} m\left(x_{k}\right)<+\infty$. Hence $\omega$ can be identified with the finite or infinite multi-subset $\left[x_{1}, x_{2}, \ldots\right]$ of $(0,1)$. We sometimes use this identification by writing $x \in \omega$ when $\omega(\{x\})>0$. The number of particles in the configuration $\omega$ is $|\omega|:=\omega(0,1) \in$ $\mathbf{Z}_{+} \cup\{+\infty\}$.

We consider $\Omega$ endowed with the weakest topology such that the map $\omega \mapsto$ $\omega(m \phi)$ is continuous for any $\phi \in C_{0}(0,1)$. Referring to Appendix A for topological amenities, we here note that for each $\ell \in \mathbf{R}_{+}$, the set $K_{\ell}:=\{\omega \in \Omega: \omega(m) \leq \ell\}$ is compact and the relative topology on $K_{\ell}$ is Polish, see Lemma A.1. Finally, we consider $\Omega$ also as a measurable space by endowing it with the Borel $\sigma$-algebra $\mathcal{B}(\Omega)$.

The dynamics will be defined, as a Markov process, by describing explicitly the transition function. We start with the case in which particles do not enter the 
system. To this end, we let $\left\{\mathbf{P}_{x}^{0}\right\}_{x \in(0,1)}$ be the Markov family of a Brownian motion on $(0,1)$ with absorption at the endpoints. We denote by $p_{t}^{0}(x, d y)$ the associated transition function and define the hitting time $\tau_{a}:=\inf \{t \geq 0: X(t)=a\}, a \in$ $[0,1]$. Set $\tau:=\tau_{0} \wedge \tau_{1}$ and observe that $m(x)=\mathbf{E}_{x}^{0}(\tau)$.

Given $\omega \in \Omega$, let $P_{t}^{0}(\omega, \cdot)$ be the law at time $t \geq 0$ of independent identical particles starting from $\omega$ and performing Brownian motions on $(0,1)$ with absorption at the endpoints. As we will show in Lemma 2.1 below, $P_{t}^{0}: \Omega \times \mathcal{B}(\Omega) \rightarrow[0,1]$, $t \geq 0$ is a time homogeneous transition function.

To describe the entrance of particles we need an auxiliary Poisson point process that we next introduce. Given a positive Radon measure $\mu$ on $(0,1)$ such that $\mu(m)<+\infty$, we denote by $\Pi_{\mu}$ the law of a Poisson point process with intensity measure $\mu$ (see e.g. Fristedt and Gray, 1997 Chapter 29). Since $\Pi_{\mu}(\omega(m))=$ $\mu(m)<+\infty$ we can regard $\Pi_{\mu}$ as a probability on $\Omega$. Given $\lambda:=\left(\lambda_{0}, \lambda_{1}\right) \in \mathbf{R}_{+}^{2}$, that will play the role of the chemical potentials of the boundary reservoirs, and $t \geq 0$ we define the Radon measure on $(0,1)$

$$
\mu_{t}^{\lambda}(d x):=\left[\lambda_{0} \mathbf{P}_{x}^{0}\left(\tau_{0} \leq t\right)+\lambda_{1} \mathbf{P}_{x}^{0}\left(\tau_{1} \leq t\right)\right] d x .
$$

The time homogeneous transition function $P_{t}$ of the boundary driven Brownian gas is defined by the following procedure. Given $\omega \in \Omega$ and $t \geq 0$ the law of $\omega(t)$ is obtained by summing two independent variables, the first sampled according to $P_{t}^{0}(\omega, \cdot)$ the second according to $\Pi_{\mu_{t}^{\lambda}}$. In other words, we let $P_{t}: \Omega \times \mathcal{B}(\Omega) \rightarrow[0,1]$, $t \geq 0$ be defined by

$$
P_{t}(\omega, B):=\iint_{\eta_{1}+\eta_{2} \in B} \Pi_{\mu_{t}^{\lambda}}\left(d \eta_{1}\right) P_{t}^{0}\left(\omega, d \eta_{2}\right) .
$$

Lemma 2.1. For each $\lambda \in \mathbf{R}_{+}^{2}$ the family $P_{t}, t \geq 0$ is a time homogeneous transition function on $(\Omega, \mathcal{B}(\Omega))$.

Remark 2.2. We observe that $P_{t}$ is not Feller. Let indeed $\omega_{n}:=n \delta_{1 / n}$, then $\omega_{n} \rightarrow$ 0 but $P_{t}\left(\omega_{n}, \cdot\right)$ does not converge to $P_{t}(0, \cdot)$. Considering in fact $n$ independent Brownian motions with absorption starting at the point $1 / n$, at time $t>0$ at least one reaches $(1 / 4,3 / 4)$ with probability uniformly bounded away from 0 .

Proof of Lemma 2.1: Plainly, $P_{0}(\omega, \cdot)=\delta_{\omega}$. Moreover, by Lemma A.4, for each $t>0$ and $\omega \in \Omega, P_{t}(\omega, \cdot)$ is a probability measure on $(\Omega, \mathcal{B}(\Omega))$. Furthermore by Lemma B.1, for each $B \in \mathcal{B}(\Omega)$ the map $\mathbf{R}_{+} \times \Omega \ni(t, \omega) \mapsto P_{t}(\omega, B)$ is Borel.

It remains to show that $P_{t}$ satisfies the Chapman-Kolmogorov equation

$$
P_{s+t}(\omega, B)=\int P_{s}(\omega, d \eta) P_{t}(\eta, B) \quad t, s \geq 0, \omega \in \Omega, B \in \mathcal{B}(\Omega) .
$$

By Lemma A.3 it is enough to show that for each $\psi \in C_{K}(0,1)$, and $s, t \geq 0$

$$
\int P_{s+t}(\omega, d \eta) e^{i \eta(\psi)}=\iint P_{s}(\omega, d \eta) P_{t}(\eta, d \zeta) e^{i \zeta(\psi)} .
$$

By definition, the left hand side of (2.3) is

$$
\begin{aligned}
& \int \Pi_{\mu_{s+t}^{\lambda}}\left(d \eta_{1}\right) \int P_{s+t}^{0}\left(\omega, d \eta_{2}\right) e^{i\left(\eta_{1}+\eta_{2}\right)(\psi)} \\
& =\exp \left\{\mu_{s+t}^{\lambda}\left(e^{i \psi}-1\right)\right\} \int P_{s+t}^{0}\left(\omega, d \eta_{2}\right) e^{i \eta_{2}(\psi)},
\end{aligned}
$$


while its right hand side is

$$
\begin{aligned}
& \int \Pi_{\mu_{s}^{\lambda}}\left(d \eta_{1}\right) \int P_{s}^{0}\left(\omega, d \eta_{2}\right) \int \Pi_{\mu_{t}^{\lambda}}\left(d \zeta_{1}\right) \int P_{t}^{0}\left(\eta_{1}+\eta_{2}, d \zeta_{2}\right) e^{i\left(\zeta_{1}+\zeta_{2}\right)(\psi)} \\
& =\exp \left\{\mu_{t}^{\lambda}\left(e^{i \psi}-1\right)\right\} \int \Pi_{\mu_{s}^{\lambda}}\left(d \eta_{1}\right) \int P_{s}^{0}\left(\omega, d \eta_{2}\right) \int P_{t}^{0}\left(\eta_{1}+\eta_{2}, d \zeta_{2}\right) e^{i \zeta_{2}(\psi)} .
\end{aligned}
$$

In view of the product structure of $P_{t}^{0}$ we get

$$
\int P_{t}^{0}\left(\eta_{1}+\eta_{2}, d \zeta_{2}\right) e^{i \zeta_{2}(\psi)}=\int P_{t}^{0}\left(\eta_{1}, d \zeta_{21}\right) e^{i \zeta_{21}(\psi)} \int P_{t}^{0}\left(\eta_{2}, d \zeta_{22}\right) e^{i \zeta_{22}(\psi)}
$$

Using the Chapman-Kolmogorov equation for $P_{t}^{0}$ (this follows easily from its product structure and the Chapman-Kolmogorov equation for $p_{t}^{0}$ )

$$
\int P_{s}^{0}\left(\omega, d \eta_{2}\right) \int P_{t}^{0}\left(\eta_{2}, d \zeta_{22}\right) e^{i \zeta_{22}(\psi)}=\int P_{s+t}^{0}\left(\omega, d \eta_{2}\right) e^{i \eta_{2}(\psi)}
$$

By the previous identities, the proof of (2.3) is completed once we show that

$$
\int \Pi_{\mu_{s}^{\lambda}}\left(d \eta_{1}\right) \int P_{t}^{0}\left(\eta_{1}, d \zeta_{21}\right) e^{i \zeta_{21}(\psi)}=\exp \left\{\mu_{s+t}^{\lambda}\left(e^{i \psi}-1\right)-\mu_{t}^{\lambda}\left(e^{i \psi}-1\right)\right\}
$$

The product structure of $P_{t}^{0}$ and standard properties of Poisson processes yield

$$
\int \Pi_{\mu_{s}^{\lambda}}\left(d \eta_{1}\right) \int P_{t}^{0}\left(\eta_{1}, d \zeta_{21}\right) e^{i \zeta_{21}(\psi)}=\exp \left\{\int \mu_{s}^{\lambda}(d x) \int p_{t}^{0}(x, d y)\left(e^{i \psi(y)}-1\right)\right\} .
$$

Thus (2.4) is implied by

$$
\int \mu_{s}^{\lambda}(d x) p_{t}^{0}(x, \cdot)=\mu_{s+t}^{\lambda}-\mu_{t}^{\lambda}
$$

We write

$$
p_{t}^{0}(x, d y)=\mathbf{P}_{x}^{0}\left(\tau_{0} \leq t\right) \delta_{0}(d y)+\mathbf{P}_{x}^{0}\left(\tau_{1} \leq t\right) \delta_{1}(d y)+q_{t}^{0}(x, y) d y
$$

and observe that $q_{t}^{0}$ is a symmetric function on $(0,1)^{2}$.

$$
\begin{aligned}
\int \mu_{s}^{\lambda}(d x) q_{t}^{0}(x, y) & =\lambda_{0} \int d x \mathbf{P}_{x}^{0}\left(\tau_{0} \leq s\right) q_{t}^{0}(x, y)+\lambda_{1} \int d x \mathbf{P}_{x}^{0}\left(\tau_{1} \leq s\right) q_{t}^{0}(x, y) \\
& =\lambda_{0} \int d x \mathbf{P}_{x}^{0}\left(\tau_{0} \leq s\right) q_{t}^{0}(y, x)+\lambda_{1} \int d x \mathbf{P}_{x}^{0}\left(\tau_{1} \leq s\right) q_{t}^{0}(y, x)
\end{aligned}
$$

By the strong Markov property of absorbed Brownian motion, for $y \in(0,1)$ :

$$
\begin{aligned}
& \mathbf{P}_{y}^{0}\left(\tau_{0} \leq s+t\right)=\mathbf{P}_{y}^{0}\left(\tau_{0} \leq t\right)+\int d x \mathbf{P}_{x}^{0}\left(\tau_{0} \leq s\right) q_{t}^{0}(y, x) \\
& \mathbf{P}_{y}^{0}\left(\tau_{1} \leq s+t\right)=\mathbf{P}_{y}^{0}\left(\tau_{1} \leq t\right)+\int d x \mathbf{P}_{x}^{0}\left(\tau_{1} \leq s\right) q_{t}^{0}(y, x)
\end{aligned}
$$

and (2.5) follows.

Lemma 2.1 yields the existence of a Markov family with transition function $P_{t}$. This however does not give any regularity of the paths. As we next state the paths of the boundary driven Brownian gas are continuous. The proof will be achieved by considering the Poissonian limit of independent sticky Brownian motions and it is deferred to Section 4.

Theorem 2.3. Given $\lambda \in \mathbf{R}_{+}^{2}$, there exists a Markov family $\left\{\mathbb{P}_{\omega}\right\}_{\omega \in \Omega}$ on $C\left(\mathbf{R}_{+}, \Omega\right)$ with transition function $P_{t}$. 
In the rest of this section we discuss some basic properties of $\mathbb{P}_{\omega}$. We start by describing its stationary measure. To this end, we premise few more definitions. Set $\bar{\lambda}(x):=\lambda_{0}(1-x)+\lambda_{1} x, x \in(0,1)$ and let $\left\{\mathbf{Q}_{x}^{0}\right\}_{x \in(0,1)}$ be the Markov family of diffusions on $(0,1)$ with absorption at the endpoints, $\operatorname{drift}(\log \bar{\lambda})^{\prime}$ and diffusion coefficient one. We denote by $g_{t}^{0}(x, d y)$ the associated time homogeneous transition function. Given $\omega \in \Omega$, let $Q_{t}^{0}(\omega, \cdot)$ be the law at time $t \geq 0$ of independent particles starting from $\omega$ and evolving according to $\mathbf{Q}^{0}$.

Let also $\nu_{t}^{\lambda}, t \in \mathbf{R}_{+}$, the measure on $(0,1)$ defined by

$$
\nu_{t}^{\lambda}(d x):=\bar{\lambda}(x) \mathbf{P}_{x}^{0}(\tau \leq t) d x .
$$

Finally, let $Q_{t}(\omega, \cdot), t \geq 0, \omega \in \Omega$ be the family of probabilities on $\Omega$ defined by

$$
Q_{t}(\omega, B):=\iint_{\eta_{1}+\eta_{2} \in B} \Pi_{\nu_{t}^{\lambda}}\left(d \eta_{1}\right) Q_{t}^{0}\left(\omega, d \eta_{2}\right) .
$$

As in Lemma 2.1, $Q_{t}$ is a time homogeneous transition function on $(\Omega, \mathcal{B}(\Omega))$.

Proposition 2.4. The distribution $\Pi_{\bar{\lambda}}$ of a Poisson point process with intensity $\bar{\lambda}(x) d x$ is the unique stationary distribution for the Markov family $\left\{\mathbb{P}_{\omega}\right\}_{\omega \in \Omega}$ and for each initial datum $\omega \in \Omega$ the law of $\omega(t)$ converges to $\Pi_{\bar{\lambda}}$. The probability $\Pi_{\bar{\lambda}}$ is reversible if and only if $\lambda_{0}=\lambda_{1}$. Furthermore, letting $T_{t}, t \geq 0$, be the semigroup on $L^{2}\left(\Omega ; \Pi_{\bar{\lambda}}\right)$ associated to the family $\left\{\mathbb{P}_{\omega}\right\}_{\omega \in \Omega}$ then its adjoint $T_{t}^{*}$ is the semigroup associated to the Markov family with transition function $Q_{t}$ given by (2.7).

Proof: Fix $\omega \in \Omega$. By the representation (2.2), $\omega(t)=\eta_{1}(t)+\eta_{2}(t)$ where $\eta_{1}(t)$ is a Poisson point process with intensity $\mu_{t}^{\lambda}$ as in (2.1) and the distribution of $\eta_{2}(t)$ is given by $P_{t}^{0}(\omega, \cdot)$. Since

$$
\int P_{t}^{0}(\omega, d \eta)|\eta|=\sum_{x \in \omega} \mathbf{P}_{x}^{0}(\tau \leq t) \leq \frac{1}{t} \sum_{x \in \omega} \mathbf{E}_{x}^{0}(\tau)=\frac{\omega(m)}{t}
$$

$\eta_{2}(t) \rightarrow 0$ in probability as $t \rightarrow+\infty$. Moreover, since $\mu_{t}^{\lambda} \rightarrow \bar{\lambda}(x) d x, \eta_{1}(t)$ converges in law to $\Pi_{\bar{\lambda}}$. This implies that $\Pi_{\bar{\lambda}}$ is the unique stationary distribution.

It remains to show that for each $f, g \in L^{2}\left(\Omega, \Pi_{\bar{\lambda}}\right)$ and $t \geq 0$,

$$
\Pi_{\bar{\lambda}}\left(g T_{t} f\right)=\Pi_{\bar{\lambda}}\left(f T_{t}^{*} g\right) .
$$

By linearity and density, it suffices to consider the case in which $f(\omega)=\exp \{i \omega(\phi)\}$ and $g(\omega)=\exp \{i \omega(\psi)\}$ for some $\phi, \psi \in C_{K}(0,1)$. That is

$$
\iint \Pi_{\bar{\lambda}}(d \omega) P_{t}(\omega, d \eta) e^{i \omega(\psi)} e^{i \eta(\phi)}=\iint \Pi_{\bar{\lambda}}(d \omega) Q_{t}(\omega, d \eta) e^{i \omega(\phi)} e^{i \eta(\psi)} .
$$

By the very definition (2.2) and standard properties of Poisson point process, the left hand side of (2.9) is equal to

$$
\exp \left\{\mu_{t}^{\lambda}\left(e^{i \phi}-1\right)-\int \bar{\lambda}(x) d x+\iint \bar{\lambda}(x) d x p_{t}^{0}(x, d y) e^{i \psi(x)} e^{i \phi(y)}\right\},
$$

while, by (2.7), the right side is equal to

$$
\exp \left\{\nu_{t}^{\lambda}\left(e^{i \psi}-1\right)-\int \bar{\lambda}(x) d x+\iint \bar{\lambda}(x) d x g_{t}^{0}(x, d y) e^{i \phi(x)} e^{i \psi(y)}\right\} .
$$


Hence, (2.9) is achieved by showing that

$$
\begin{aligned}
& \iint \bar{\lambda}(x) d x p_{t}^{0}(x, d y) e^{i \psi(x)} e^{i \phi(y)} \\
& \quad=\nu_{t}^{\lambda}\left(e^{i \psi}\right)-\mu_{t}^{\lambda}\left(e^{i \phi}\right)+\iint \bar{\lambda}(x) d x g_{t}^{0}(x, d y) e^{i \phi(x)} e^{i \psi(y)}
\end{aligned}
$$

and

$$
\mu_{t}^{\lambda}(1)=\nu_{t}^{\lambda}(1)
$$

Simple computations shows that the transition function $g_{t}^{0}$ of the Markov family $\mathbf{Q}_{x}^{0}$ satisfies

$$
g_{t}^{0}(x, d y)=\frac{\bar{\lambda}(y)}{\bar{\lambda}(x)} p_{t}^{0}(x, d y),
$$

so that (2.10) follows straightforwardly.

To prove (2.11), since it trivially holds for $t=0$, it suffices to show that $\frac{d}{d t}\left[\mu_{t}^{\lambda}(1)-\nu_{t}^{\lambda}(1)\right]=0$. By using that $\mathbf{P}_{x}^{0}(\tau \leq t)=\mathbf{P}_{x}^{0}\left(\tau_{0} \leq t\right)+\mathbf{P}_{x}^{0}\left(\tau_{1} \leq t\right)$ and that $\mathbf{P}_{x}^{0}\left(\tau_{i} \leq t\right)$ solves the heat equation, an integration by parts and the symmetry $\mathbf{P}_{x}^{0}\left(\tau_{0} \leq t\right)=\mathbf{P}_{1-x}^{0}\left(\tau_{1} \leq t\right)$ yield the claim.

The next statement confirms the heuristic picture of the boundary driven Brownian gas of infinitely many particles entering and leaving the system in each time interval. On the other hand, at any fixed positive time there are only finitely many particles in the system.

Proposition 2.5. Let $\omega \in \Omega$ and $t>0$. Then $\mathbb{P}_{\omega}(|\omega(t)|<+\infty)=1$ while $\mathbb{P}_{\omega}\left(\sup _{s \in[0, t]}|\omega(s)|<+\infty\right)=0$.

Proof: Observe that the map $\Omega \ni \omega \mapsto|\omega| \in \mathbf{Z}_{+} \cup\{+\infty\}$ is Borel as pointwise limit of continuous maps. The first statement follows from the stronger property $\mathbb{E}_{\omega}(|\omega(t)|)<+\infty$. In fact, by the very definitions (2.1) and (2.2),

$$
\mathbb{E}_{\omega}(|\omega(t)|)=\int \Pi_{\mu_{t}^{\lambda}}(d \eta)|\eta|+\int P_{t}^{0}(\omega, d \eta)|\eta|=\lambda_{0}+\lambda_{1}+\frac{\omega(m)}{t},
$$

where we used (2.8).

The proof of the second statement is split in few steps.

Step 1. If $t>0$ then $\mathbb{P}_{0}(|\omega(s)|=0, \forall s \in[0, t])=0$.

Let us observe that the map $\Omega \ni \omega \mapsto \omega\left(m^{2}\right) \in \mathbf{R}$ is continuous and $|\omega|=0$ if and only if $\omega\left(m^{2}\right)=0$. Since the evaluation map $C([0,+\infty) ; \Omega) \ni \omega \mapsto \omega(s) \in \Omega$ is continuous, the condition $s \in[0, t]$ can be replaced by $s \in[0, t] \cap \mathbf{Q}$. Denote by $Q_{n}$ the set of points in $[0, t]$ of the form $k / n$, for some $k \in \mathbf{N}$. Then by the Markov property and (2.2)

$$
\begin{aligned}
& \mathbb{P}_{0}(|\omega(s)|=0, \forall s \in[0, t])=\lim _{n \rightarrow+\infty} \mathbb{P}_{0}\left(|\omega(s)|=0, \forall s \in Q_{n}\right) \\
& =\lim _{n \rightarrow+\infty} \prod_{s \in Q_{n}} P_{1 / n}(0,\{0\})=\lim _{n \rightarrow+\infty} \Pi_{\mu_{1 / n}^{\lambda}}(\{0\})^{\lfloor n t\rfloor} \\
& =\lim _{n \rightarrow+\infty} \exp \left\{-\lfloor n t\rfloor \mu_{1 / n}^{\lambda}(0,1)\right\} .
\end{aligned}
$$

As simple to check, $\lim _{s \downarrow 0} \sqrt{s} \mu_{s}^{\lambda}(0,1)>0$ which concludes the proof of this step. Step 2. Let $\eta_{1}, \eta_{2}$ be two independent processes with distribution $\mathbb{P}_{\omega_{i}}$ with parameter $\lambda^{i}, i=1,2$. Then the distribution of the process $\eta_{1}+\eta_{2}$ is $\mathbb{P}_{\omega_{1}+\omega_{2}}$ with parameter $\lambda^{1}+\lambda^{2}$. 
The proof amounts to a straightforward computation that is omitted. Step 3. If $0 \leq a<b$ and $\omega \in \Omega$, then $\mathbb{P}_{\omega}(\exists s \in[a, b]:|\omega(s)|>0)=1$.

By Step 2, the process starting from 0 is stochastically dominated, in the sense of Radon measures, by the one starting from $\omega$. Thus, by Step 1 , if $t>0$ then $\mathbb{P}_{\omega}(\exists s \in[0, t]:|\omega(s)|>0)=1$. By the Markov property

$$
\mathbb{P}_{\omega}\left(\sup _{s \in[a, b]}|\omega(s)|>0\right)=\int P_{a}(\omega, d \eta) \mathbb{P}_{\eta}\left(\sup _{s \in[0, b-a]}|\omega(s)|>0\right)=1 .
$$

Conclusion. If $t>0$ and $\ell \in \mathbf{N}$, then $\mathbb{P}_{\omega}\left(\sup _{s \in[0, t]}|\omega(s)| \geq \ell\right)=1$.

In view of Step 2 it suffices to consider the case $\omega=0$. Again by Step 2 the process with law $\mathbb{P}_{0}$ and parameter $\lambda$ can be realized as the sum of $\ell$ independent and identically distributed processes $\omega^{k}, k=1, \ldots, \ell$, with law $\mathbb{P}_{0}$ and parameter $\lambda / \ell$. Denote by $\mathcal{I}$ the collection of intervals in $[0, t]$ with rational endpoints. In view of Step 3, with probability one for each $[a, b] \in \mathcal{I}$ there exists $s \in[a, b]$ such that $\left|\omega^{k}(s)\right| \geq 1$. We next observe that for each $\omega \in C([0,+\infty) ; \Omega)$ the set $\{t:|\omega(t)|>0\}$ is an open subset of $[0,+\infty)$. Indeed, its complement is the zero level set of the continuous map $t \mapsto \omega(t)\left(m^{2}\right)$.

By the previous observations, on a set of probability one there exist $s_{1} \in[0, t]$ such that $\left|\omega^{1}\left(s_{1}\right)\right| \geq 1$ and $I_{1} \in \mathcal{I}, s_{1} \in I_{1}$ such that $\left|\omega^{1}(s)\right| \geq 1$ for all $s \in I_{1}$. Next, again with probability one, there exist $s_{2} \in I_{1}$ such that $\left|\omega^{2}\left(s_{2}\right)\right| \geq 1$ and $I_{2} \in \mathcal{I}, s_{2} \in I_{2} \subset I_{1}$ such that $\left|\omega^{1}(s)+\omega^{2}(s)\right| \geq 2$ for all $s \in I_{2}$. By iterating this procedure we conclude the proof.

\section{Other constructions and empirical flow}

In this section we present two alternative constructions of the boundary driven Brownian gas. We then define, by a suitable limiting procedure, the empirical flow that counts the net amount of particles crossing a given point and describe explicitly its statistics.

3.1. Construction from the excursion process. A positive excursion of the Brownian motion is the part of the path $B(t), t \in\left[t_{1}, t_{2}\right]$ such that $B\left(t_{1}\right)=B\left(t_{2}\right)=0$ and $B(t)>0$ for $t \in\left(t_{1}, t_{2}\right)$. The excursion process describes the statistics of such excursions; the Brownian motion can be recovered from it by gluing, according to the local time, different excursions, see e.g. $§ 5.15$ of Varadhan (2007). As we next show, the boundary driven Brownian gas can be naturally realized from the excursion process of a single Brownian motion. Since we consider the boundary driven Brownian gas on a bounded interval, we need first to introduce the excursion process for a Brownian motion with absorption at the end points. We remark however that had we considered the boundary driven Brownian gas on the positive half line, we would have only needed the excursion process of a standard Brownian motion.

The excursion process for a Brownian motion on $[0,1]$ with absorption at the end-points is defined as follows. Let $\wp$ be the $\sigma$-finite measure on $(0,+\infty)$ defined by

$$
\wp(d \ell)=\lim _{\varepsilon \downarrow 0} \frac{1}{\varepsilon} \mathbf{P}_{\varepsilon}^{0}(\tau \in d \ell)=\lim _{\varepsilon \downarrow 0} \frac{1}{\varepsilon} \mathbf{P}_{1-\varepsilon}^{0}(\tau \in d \ell) .
$$


Let also $\mathbf{P}^{0, \ell}$ be the law of a right excursion from 0 of length $\ell$ and $\mathbf{P}^{1, \ell}$ be the law of a left excursion from 1 of length $\ell$, that is,

$$
\mathbf{P}^{0, \ell}=\lim _{\varepsilon \downarrow 0} \mathbf{P}_{\varepsilon}^{0}(\cdot \mid \tau=\ell) \quad \mathbf{P}^{1, \ell}=\lim _{\varepsilon \downarrow 0} \mathbf{P}_{1-\varepsilon}^{0}(\cdot \mid \tau=\ell) .
$$

To define the excursion process we define the sets

$$
\begin{aligned}
& \mathcal{E}_{0}:=\{(\ell, X), \ell \in(0,+\infty), X \in C([0, \ell] ;[0,1]): \\
&X(0)=0, X(\ell) \in\{0,1\}, X(t) \in(0,1), t \in(0, \ell)\}
\end{aligned}
$$

and

$$
\begin{aligned}
\mathcal{E}_{1}:=\{(\ell, X), \ell \in(0,+\infty), X \in C([0, \ell] ;[0,1]): & \\
& X(0)=1, X(\ell) \in\{0,1\}, X(t) \in(0,1), t \in(0, \ell)\} .
\end{aligned}
$$

The excursion process, for the Brownian motion with absorption at the end-points is given by two independent Poisson point processes $\left\{\left(s_{j}^{i}, \ell_{j}^{i}, X_{j}^{i}\right)\right\}_{j \in \mathbf{N}}$ on $[0,+\infty) \times \mathcal{E}_{i}$ with intensity measures $\left(\lambda_{i} / 2\right) d s \wp(d \ell) d \mathbf{P}^{i, \ell}, i=0,1$.

Given $\Omega \ni \omega=\sum_{k} \delta_{x_{k}}$, let $B_{k}^{0}$ be $|\omega|$ independent Brownian motions on $[0,1]$ with absorption at the end-points starting from $x_{k}, k=1, \ldots,|\omega|$. Let finally $\zeta(t)$, $t \geq 0$ be the process defined by

$$
\zeta(t):=\sum_{k} \delta_{B_{k}^{0}(t)}+\sum_{j: 0 \leq t-s_{j}^{0} \leq \ell_{j}^{0}} \delta_{X_{j}^{0}\left(t-s_{j}^{0}\right)}+\sum_{j: 0 \leq t-s_{j}^{1} \leq \ell_{j}^{1}} \delta_{X_{j}^{1}\left(t-s_{j}^{1}\right)},
$$

that we regard as a random measure on $(0,1)$ understanding that if $x \in\{0,1\}$ then $\delta_{x}$ gives no weight to the right hand side of (3.1).

Theorem 3.1. The law of the process $\zeta$ is $\mathbb{P}_{\omega}$.

Proof: By standard properties of Poisson processes, $\zeta$ is Markovian. We next identify its transition function by showing that for each $t>0$ and $\omega \in \Omega$

$$
\zeta(t) \stackrel{\text { Law }}{=} P_{t}(\omega, \cdot)
$$

where the right hand side is defined in (2.2). In view of the independence, (2.1), standard properties of the Poisson process, and Lemma A.3 it is enough to show that for each $\psi \in C_{K}(0,1)$

$$
\begin{aligned}
& \frac{1}{2} \int_{0}^{t} d s \int_{t-s}^{+\infty} \wp(d \ell) \mathbf{E}^{0, \ell}\left[e^{i \psi(X(t-s))}-1\right]=\int_{0}^{1} d x \mathbf{P}_{x}^{0}\left(\tau_{0} \leq t\right)\left[e^{i \psi(x)}-1\right] \\
& \frac{1}{2} \int_{0}^{t} d s \int_{t-s}^{+\infty} \wp(d \ell) \mathbf{E}^{1, \ell}\left[e^{i \psi(X(t-s))}-1\right]=\int_{0}^{1} d x \mathbf{P}_{x}^{0}\left(\tau_{1} \leq t\right)\left[e^{i \psi(x)}-1\right] .
\end{aligned}
$$

We prove the first equation. By a change of variables it is equivalent to

$$
\frac{1}{2} \int_{t}^{+\infty} \wp(d \ell) \mathbf{P}^{0, \ell}(X(t) \in d x)=\frac{d}{d t} \mathbf{P}_{x}^{0}\left(\tau_{0} \leq t\right) d x .
$$

We next observe that for any $\varepsilon \in(0,1)$

$$
\mathbf{P}_{\varepsilon}^{0}(X(t) \in d x)=\int_{t}^{+\infty} \mathbf{P}_{\varepsilon}^{0}(\tau \in d \ell) \mathbf{P}_{\varepsilon}^{0}(X(t) \in d x \mid \tau=\ell) .
$$


Therefore

$$
\int_{t}^{+\infty} \wp(d \ell) \mathbf{P}^{0, \ell}(X(t) \in d x)=\lim _{\varepsilon \downarrow 0} \frac{1}{\varepsilon} \mathbf{P}_{\varepsilon}^{0}(X(t) \in d x) .
$$

As in (2.6), let $q_{t}^{0}$ be the density of the absolutely continuous part of the transition probability of the Brownian motion with absorption at the endpoints. The proof of (3.3) is achieved by showing

$$
\lim _{\varepsilon \downarrow 0} \frac{1}{2 \varepsilon} q_{t}^{0}(\varepsilon, x)=\frac{d}{d t} \mathbf{P}_{x}^{0}\left(\tau_{0} \leq t\right), \quad t>0 .
$$

This identity can be checked by comparing the explicit expression for the right hand side of (2.1.4) (1) in Borodin and Salminen (2002) with the representation of the left hand side obtained by the image method.

3.2. Graphical construction. Since in any time interval infinitely many particles enters from the reservoirs, a full graphical construction of the boundary driven Brownian gas does not appear feasible. It is however possible to provide a graphical construction for the restriction of the process to the interval $[a, 1-a] \subset(0,1)$. We next discuss such graphical construction for the stationary process only.

Let $N^{0}=\left\{\sigma_{k}^{0}\right\}_{k \in \mathbf{Z}}$ and $N^{1}=\left\{\sigma_{k}^{1}\right\}_{k \in \mathbf{Z}}$ be two independent Poisson point processes on $\mathbf{R}$ with intensity $\lambda_{0} /(2 a)$ and $\lambda_{1} /(2 a)$ respectively. At each time in $\sigma_{k}^{0} \in N^{0}$ (respectively $\sigma_{k}^{0} \in N^{1}$ ) we let $\left\{B_{k}^{0}(t)\right\}_{t \geq \sigma_{k}^{0}}$ (respectively $\left\{B_{k}^{1}(t)\right\}_{t \geq \sigma_{k}^{1}}$ ) be a Brownian motion on $(0,1)$ with absorption at the endpoints and initial datum $B_{k}^{0}\left(\sigma_{k}^{0}\right)=a$ (respectively $B_{k}^{1}\left(\sigma_{k}^{1}\right)=1-a$ ). All these Brownian motions are independent and independent from the Poisson point processes. We define

$$
\omega^{a}(t):=\sum_{k: \sigma_{k}^{0} \leq t} \delta_{B_{k}^{0}(t)}+\sum_{k: \sigma_{k}^{1} \leq t} \delta_{B_{k}^{1}(t)}, \quad t \in \mathbf{R} .
$$

By standard properties of Poisson processes, $\sup _{t \in \mathbf{R}}\left|\omega^{a}(t)\right|<+\infty$ a.s. The law of $\omega^{a}$ is denoted by $\mathbb{P}^{a}$ that we consider as a probability on $C(\mathbf{R}, \Omega)$. Let also $\Omega^{a}$ be the set of integer valued Radon mesures on $[a, 1-a]$ and observe that $\Omega^{a}$ is naturally embedded in $\Omega$.

Theorem 3.2. Fix $\lambda \in \mathbf{R}_{+}^{2}$ and let $\mathbb{P}_{\Pi_{\bar{\lambda}}}$ be the stationary process associated to the Markov family $\left\{\mathbb{P}_{\omega}\right\}_{\omega \in \Omega}$. The restrictions of $\mathbb{P}^{a}$ and $\mathbb{P}_{\Pi_{\bar{\lambda}}}$ to $C\left(\mathbf{R}, \Omega^{a}\right)$ coincide.

We notice that this graphical construction implies a Burke type theorem for the boundary driven Brownian gas, see Ferrari and Fontes (1994), for a discussion about Burke theorem in the context of interacting particles systems on the lattice. For instance, in the reversible case $\lambda_{0}=\lambda_{1}$, Theorem 3.2 implies the following statement. Under the stationary process, the distribution of the times of last visit of the point $a \in(0,1 / 2)$ is a Poisson point process of parameter $\lambda_{0} /(2 a)$.

Proof of Theorem 3.2: By Lemma A.5 it is enough to show that the finite dimensional distributions of the restriction to $C\left(\mathbf{R}, \Omega^{a}\right)$ of $\mathbb{P}^{a}$ and $\mathbb{P}_{\Pi_{\bar{\lambda}}}$ coincide. By Lemma A.3 it is enough to show that for each $t_{1}<\cdots<t_{n}$ and each $\psi_{1}, \ldots, \psi_{n}$ : $(0,1) \rightarrow \mathbf{R}$, bounded mesurable and vanishing on $(0,1) \backslash[a, 1-a]$,

$$
\mathbb{E}^{a}\left[\exp \left\{i \sum_{j=1}^{n} \omega\left(t_{j}\right)\left(\psi_{j}\right)\right\}\right]=\mathbb{E}_{\Pi_{\bar{\lambda}}}\left[\exp \left\{i \sum_{j=1}^{n} \omega\left(t_{j}\right)\left(\psi_{j}\right)\right\}\right] .
$$


To keep combinatorics simple we discuss only the case $n=2$. By standard properties of Poisson point processes,

$$
\begin{aligned}
& \log \mathbb{E}^{a}\left[e^{i \omega\left(t_{1}\right)\left(\psi_{1}\right)+i \omega\left(t_{2}\right)\left(\psi_{2}\right)}\right] \\
& =\frac{\lambda_{0}}{2 a}\left[\int_{-\infty}^{t_{1}} d t \iint p_{t_{1}-t}^{0}\left(a, d x_{1}\right) p_{t_{2}-t_{1}}^{0}\left(x_{1}, d x_{2}\right)\left(e^{i \psi_{1}\left(x_{1}\right)+i \psi_{2}\left(x_{2}\right)}-1\right)\right. \\
& \left.\quad+\int_{t_{1}}^{t_{2}} d t \int p_{t_{2}-t}^{0}\left(a, d x_{2}\right)\left(e^{i \psi_{2}\left(x_{2}\right)}-1\right)\right] \\
& +\frac{\lambda_{1}}{2 a}\left[\int_{-\infty}^{t_{1}} d t \iint p_{t_{1}-t}^{0}\left(1-a, d x_{1}\right) p_{t_{2}-t_{1}}^{0}\left(x_{1}, d x_{2}\right)\left(e^{i \psi_{1}\left(x_{1}\right)+i \psi_{2}\left(x_{2}\right)}-1\right)\right. \\
& \left.\quad+\int_{t_{1}}^{t_{2}} d t \int p_{t_{2}-t}^{0}\left(1-a, d x_{2}\right)\left(e^{i \psi_{2}\left(x_{2}\right)}-1\right)\right]
\end{aligned}
$$

Recall (2.6) and observe that $(1 / 2) \int_{0}^{\infty} d t q_{t}^{0}(x, y)$ is the Green function of the Dirichlet Laplacian on $(0,1)$, i.e.

$$
\int_{0}^{\infty} d t q_{t}^{0}(x, y)= \begin{cases}2 x(1-y) & \text { if } x \leq y \\ 2(1-x) y & \text { if } x>y\end{cases}
$$

By writing

$$
e^{i \psi_{1}\left(x_{1}\right)+i \psi_{2}\left(x_{2}\right)}-1=\left(e^{i \psi_{1}\left(x_{1}\right)}-1\right)\left(e^{i \psi_{2}\left(x_{2}\right)}-1\right)+e^{i \psi_{1}\left(x_{1}\right)}-1+e^{i \psi_{2}\left(x_{2}\right)}-1
$$

and using that $e^{i \psi_{j}}-1$ vanishes on $(0,1) \backslash(a, 1-a)$ together with the ChapmanKolmogorov equation for $\left\{p_{t}^{0}\right\}_{t \geq 0}$ and (3.5), few computations yield

$$
\begin{aligned}
& \log \mathbb{E}^{a}\left[e^{i \omega\left(t_{1}\right)\left(\psi_{1}\right)+i \omega\left(t_{2}\right)\left(\psi_{2}\right)}\right] \\
& =\int d x \bar{\lambda}(x)\left\{e^{i \psi_{1}(x)}+e^{i \psi_{2}(x)}-2+\left(e^{i \psi_{1}(x)}-1\right) \int d y q_{t_{2}-t_{1}}^{0}(x, y)\left(e^{i \psi_{2}(y)}-1\right)\right\}
\end{aligned}
$$

where we recall that $\bar{\lambda}(x)=\lambda_{0}(1-x)+\lambda_{1} x$. Observe in particular that the right hand side does not depend on $a$.

On the other hand, by using (2.2)

$$
\begin{aligned}
& \mathbb{E}_{\Pi_{\bar{\lambda}}}\left[\exp \left\{i \omega\left(t_{1}\right)\left(\psi_{1}\right)+i \omega\left(t_{2}\right)\left(\psi_{2}\right)\right\}\right] \\
& =\iiint \Pi_{\bar{\lambda}}\left(d \eta_{1}\right) \Pi_{\mu_{t_{2}-t_{1}}^{\lambda}}\left(d \eta_{21}\right) P_{t_{2}-t_{1}}^{0}\left(\eta_{1}, d \eta_{22}\right) e^{i\left(\eta_{1}\left(\psi_{1}\right)+\eta_{21}\left(\psi_{2}\right)+\eta_{22}\left(\psi_{2}\right)\right)} \\
& =\exp \left\{\mu_{t_{2}-t_{1}}^{\lambda}\left(e^{i \psi_{2}}-1\right)+\iint d x_{1} \bar{\lambda}\left(x_{1}\right) p_{t_{2}-t_{1}}^{0}\left(x_{1}, d x_{2}\right)\left[e^{i \psi_{1}\left(x_{1}\right)+i \psi_{2}\left(x_{2}\right)}-1\right]\right\}
\end{aligned}
$$

whence, using again (3.6),

$$
\begin{aligned}
& \log \mathbb{E}_{\Pi_{\bar{\lambda}}}\left[\exp \left\{i \omega\left(t_{1}\right)\left(\psi_{1}\right)+i \omega\left(t_{2}\right)\left(\psi_{2}\right)\right\}\right] \\
& =\int d x \bar{\lambda}(x)\left\{e^{i \psi_{1}(x)}+e^{i \psi_{2}(x)}-2+\left(e^{i \psi_{1}(x)}-1\right) \int d y q_{t_{2}-t_{1}}^{0}(x, y)\left(e^{i \psi_{2}(y)}-1\right)\right\} \\
& \quad+R\left(\psi_{2}\right)
\end{aligned}
$$


where

$$
\begin{aligned}
R\left(\psi_{2}\right):= & \mu_{t_{2}-t_{1}}^{\lambda}\left(e^{i \psi_{2}}-1\right)-\int d x \bar{\lambda}(x)\left(e^{i \psi_{2}(x)}-1\right) \\
& +\iint d x_{1} d x_{2} \bar{\lambda}\left(x_{1}\right) q_{t_{2}-t_{1}}^{0}\left(x_{1}, x_{2}\right)\left(e^{i \psi_{2}\left(x_{2}\right)}-1\right) .
\end{aligned}
$$

It remains to show that $R\left(\psi_{2}\right)=0$. Recalling (2.1), set

$$
u(t, x):=\lambda_{0} \mathbf{P}_{x}^{0}\left(\tau_{0} \leq t\right)+\lambda_{1} \mathbf{P}_{x}^{0}\left(\tau_{1} \leq t\right)-\bar{\lambda}(x)
$$

and

$$
v(t, x):=\int d y q_{t}^{0}(x, y) \bar{\lambda}(y)
$$

By using $q_{t}^{0}(x, y)=q_{t}^{0}(y, x)$, we get

$$
R\left(\psi_{2}\right)=\int d x\left[u\left(t_{2}-t_{1}, x\right)+v\left(t_{2}-t_{1}, x\right)\right]\left(e^{i \psi_{2}(x)}-1\right) .
$$

As simple to check, the function $w:=u+v$ solves the heat equation on the interval $(0,1)$ with Dirichlet boundary conditions at the endpoints and initial datum $w(0, x)=0$. Hence $w=0$.

3.3. Empirical flow. Given a point $x \in(0,1)$ and a time interval $[0, t]$, we would like to define the (integrated) empirical flow at $x$ as the difference between the number of particles that in the time interval $[0, t]$ have crossed $x$ from left to right and the ones that crossed from right to left. Due to the Brownian nature of the paths, the above naive definition is not feasible and some care is needed. Instead of the point $x$ we shall consider the small interval $(x-\varepsilon, x+\varepsilon)$ and count the number of left/right, respectively right/left, crossing of this interval. We then take the limit $\varepsilon \rightarrow 0$ obtaining a well defined real process $J^{x}(t)$ whose law will be identified for the stationary process. For $t$ large, $J^{x}(t)$ essentially behave as the difference of two independent Poisson processes of parameters $\lambda_{0} / 2$ and $\lambda_{1} / 2$.

Given $x \in(0,1)$ and $0<\varepsilon<x \wedge(1-x)$ we define the real process $J_{\varepsilon}^{x}(t)$, $t \geq 0$, according to the following algorithm. We need three collections of tokens respectively labelled $\odot, \ominus$, and $\oplus$, together an integer valued counter.

The counter is initialized at 0 and to each particle starting in $(x-\varepsilon, x+\varepsilon)$ is given a $\odot$-token (the crossings of these particles will not be accounted for).

At $x-\varepsilon$ there is a $\ominus$-booth operating with the following directives, applying to each particle crossing $x-\varepsilon$ :

- particles having no token are given a $\ominus$-token,

- particles having either $\odot$-token or a $\ominus$-token are ignored,

- particles having a $\oplus$-token are deprived of their token, given a $\ominus$-token, and the counter is decreased by one.

Analogously, at $x+\varepsilon$ there is a $\oplus$-booth operating with the following directives, applying to each particle crossing $x+\varepsilon$ :

- particles having no token are given a $\oplus$-token,

- particles having either $\odot$-token or a $\oplus$-token are ignored,

- particles having a $\ominus$-token are deprived of their token, given a $\oplus$-token, and the counter is increased by one. 
We then define $J_{\varepsilon}^{x}(t)$ as the value of the counter at time $t$. By standard properties of Brownian motion, this defines a.s. a real process $J_{\varepsilon}^{x}$.

The next result identifies the limiting law of $J_{\varepsilon}^{x}$ as $\varepsilon \rightarrow 0$. We refer to Ferrari and Fontes (1994) for a similar result in the context of interacting particles system on the lattice.

Theorem 3.3. Let the path $\omega=\omega(t)$ be sampled according to the stationary process $\mathbb{P}_{\Pi_{\bar{\lambda}}}$ and fix $x \in(0,1)$. There exists real process $J^{x}$ such that, with probability one, for any $T>0$

$$
\lim _{\varepsilon \rightarrow 0} \sup _{t \in[0, T]}\left|J_{\varepsilon}^{x}(t)-J^{x}(t)\right|=0 .
$$

Moreover,

$$
J^{x} \stackrel{\text { Law }}{=} N^{01}-N^{10}+R^{x}
$$

where $N^{01}$ and $N^{10}$ are independent Poisson processes of parameter $\lambda_{0} / 2$ and $\lambda_{1} / 2$, while $R^{x}(t)=Y^{x}(t)-Y^{x}(0)$ where $Y^{x}$ is a stationary process satisfying the following bound. There exist constants $c, \ell_{0}>0$ depending on $\lambda$ such that for any $x \in(0,1)$, $t>0$, and $\ell \geq \ell_{0}$

$$
\mathbb{P}_{\Pi_{\bar{\lambda}}}\left(\left|Y^{x}(t)\right|>\ell\right) \leq \exp \{-c \ell\} .
$$

Proof: Pick $a \in(0,1 / 2)$ such that $(x-\varepsilon, x+\varepsilon) \subset \subset(a, 1-a)$. We realize the stationary process $\omega$ in the strip $(a, 1-a)$ according to the graphical construction discussed in Section 3.2. Recalling (3.4), for $s \in[0, t]$, we write

$$
\omega^{a}(s):=\sum_{i=0}^{1} \sum_{k: \sigma_{k}^{i} \leq s} \delta_{B_{k}^{i}(s)}=\sum_{i=0}^{1}\left(\sum_{k: \sigma_{k}^{i} \leq 0} \delta_{B_{k}^{i}(s)}+\sum_{k: 0<\sigma_{k}^{i} \leq s} \delta_{B_{k}^{i}(s)}\right),
$$

where $\left\{\sigma_{k}^{0}\right\}_{k \in \mathbf{Z}},\left\{\sigma_{k}^{1}\right\}_{k \in \mathbf{Z}}$ are two independent Poisson point processes with parameters $\lambda_{0} /(2 a), \lambda_{1} /(2 a)$ and $B_{k}^{0}$ respectively $B_{k}^{1}$ are independent Brownian motions on $[0,1]$ with absorption at the end-points starting at time $\sigma_{k}^{0}$ at $a$ respectively $\sigma_{k}^{1}$ at $1-a$. As in Section 3.2, the law of $\omega^{a}$ is denoted by $\mathbb{P}^{a}$. Observe that the process $J_{\varepsilon}^{x}$ can be obtained from $\omega^{a}$ only.

By the very definition of $J_{\varepsilon}^{x}(t)$, a straightforward tokens bookkeeping yields

$$
\begin{aligned}
J_{\varepsilon}^{x}(t)= & \sum_{i=0}^{1}\left|\left\{k: \sigma_{k}^{i} \leq 0, B_{k}^{i}(0) \in(0, x-\varepsilon), B_{k}^{i}(t) \in(x+\varepsilon, 1]\right\}\right| \\
& -\sum_{i=0}^{1}\left|\left\{k: \sigma_{k}^{i} \leq 0, B_{k}^{i}(0) \in(x+\varepsilon, 1), B_{k}^{i}(t) \in[0, x-\varepsilon)\right\}\right| \\
& +\left|\left\{k: \sigma_{k}^{0} \in(0, t), B_{k}^{0}(t) \in(x+\varepsilon, 1]\right\}\right| \\
& -\left|\left\{k: \sigma_{k}^{1} \in(0, t), B_{k}^{1}(t) \in[0, x-\varepsilon)\right\}\right| .
\end{aligned}
$$

By taking the limit $\varepsilon \rightarrow 0$ we get (3.7) with

$$
\begin{aligned}
& J^{x}(t)=\sum_{i=0}^{1}\left|\left\{k: \sigma_{k}^{i} \leq 0, B_{k}^{i}(0) \in(0, x), B_{k}^{i}(t) \in(x, 1]\right\}\right| \\
& -\sum_{i=0}^{1}\left|\left\{k: \sigma_{k}^{i} \leq 0, B_{k}^{i}(0) \in(x, 1), B_{k}^{i}(t) \in[0, x)\right\}\right| \\
& +\left|\left\{k: \sigma_{k}^{0} \in(0, t), B_{k}^{0}(t) \in(x, 1]\right\}\right|-\left|\left\{k: \sigma_{k}^{1} \in(0, t), B_{k}^{1}(t) \in[0, x)\right\}\right| .
\end{aligned}
$$


We now mark the points of $\left\{\sigma_{k}^{0}\right\}_{k \in \mathbf{Z}}$ according to the absorption end-point of the Brownian motion started at time $\sigma_{k}^{0}$. Namely, we denote by $\left\{\sigma_{k}^{00}\right\}_{k \in \mathbf{Z}}$ the starting times of the Brownian motions eventually absorbed at 0 and by $\left\{\sigma_{k}^{01}\right\}_{k \in \mathbf{Z}}$ the starting times of the Brownian motions eventually absorbed at 1 . These marks are inherited by the Brownian motions starting at the times $\left\{\sigma_{k}^{0}\right\}_{k \in \mathbf{Z}}$ which will be denoted by $\left\{B_{k}^{00}\right\}_{k \in \mathbf{Z}}$ and $\left\{B_{k}^{01}\right\}_{k \in \mathbf{Z}}$. Then $\left\{\sigma_{k}^{00}\right\}_{k \in \mathbf{Z}}$ and $\left\{\sigma_{k}^{01}\right\}_{k \in \mathbf{Z}}$ are independent Poisson point processes of parameters $(1-a) \lambda_{0} /(2 a)$ and $\lambda_{0} / 2$, while $B_{k}^{00}, B_{k}^{01}$ are independent Brownian motions on $[0,1]$ with absorption at the end-points started at time $\sigma_{k}^{00}, \sigma_{k}^{01}$ in $a$ conditioned to be absorbed at 0,1 respectively. The analogous definitions and notation is used for the Poisson point processes $\left\{\sigma_{k}^{1}\right\}_{k \in \mathbf{Z}}$ and the corresponding Brownian motions.

We now set $N^{01}(t):=\left|\left\{k: \sigma_{k}^{01} \in[0, t]\right\}\right|, N^{10}(t):=\left|\left\{k: \sigma_{k}^{10} \in[0, t]\right\}\right|$ and

$$
\begin{aligned}
& R^{x}(t)=\sum_{i=0}^{1} \sum_{j=0}^{1}\left(\left|\left\{k: \sigma_{k}^{i j}<0, B_{k}^{i j}(0) \in(0, x), B_{k}^{i j}(t) \in(x, 1]\right\}\right|\right. \\
& \left.-\left|\left\{k: \sigma_{k}^{i j}<0, B_{k}^{i j}(0) \in(x, 1), B_{k}^{i j}(t) \in[0, x)\right\}\right|\right) \\
& +\left|\left\{k: \sigma_{k}^{00} \in[0, t], B_{k}^{00}(t) \in(x, 1)\right\}\right|-\left|\left\{k: \sigma_{k}^{01} \in[0, t], B_{k}^{01}(t) \in(0, x)\right\}\right| \\
& -\left|\left\{k: \sigma_{k}^{11} \in[0, t], B_{k}^{11}(t) \in(0, x)\right\}\right|+\left|\left\{k: \sigma_{k}^{10} \in[0, t], B_{k}^{10}(t) \in(x, 1)\right\}\right| .
\end{aligned}
$$

Then (3.8) holds. It remains to analyze $R^{x}$. Set

$$
\begin{aligned}
& Y_{00}^{x}(t):=\left|\left\{k: \sigma_{k}^{00} \leq t, B_{k}^{00}(t) \in(x, 1)\right\}\right| \\
& Y_{01}^{x}(t):=\left|\left\{k: \sigma_{k}^{01} \leq t, B_{k}^{01}(t) \in(0, x)\right\}\right| \\
& Y_{10}^{x}(t):=\left|\left\{k: \sigma_{k}^{10} \leq t, B_{k}^{10}(t) \in(x, 1)\right\}\right| \\
& Y_{11}^{x}(t):=\left|\left\{k: \sigma_{k}^{11} \leq t, B_{k}^{11}(t) \in(0, x)\right\}\right| .
\end{aligned}
$$

Observe that these processes are independent and, as simple to check, stationary. A straightforward computation shows that

$R^{x}(t)=\left[Y_{00}^{x}(t)-Y_{00}^{x}(0)\right]-\left[Y_{01}^{x}(t)-Y_{01}^{x}(0)\right]+\left[Y_{10}^{x}(t)-Y_{10}^{x}(0)\right]-\left[Y_{11}^{x}(t)-Y_{11}^{x}(0)\right]$, so that, by setting $Y^{x}=Y^{00}-Y^{01}-Y^{10}+Y^{11}$, it holds $R^{x}(t)=Y^{x}(t)-Y^{x}(0)$. The bound (3.9) is finally derived by computing the exponential moments of $Y_{i j}^{x}(t)$, $i, j \in\{0,1\}$ and using the exponential Chebyshev inequality.

\section{Poissonian limit of sticky Brownian motions}

In this section we obtain the boundary driven Brownian gas by considering the Poissonian limit of independent sticky Brownian motions on the interval $[0,1]$. As a byproduct of this convergence, we deduce the continuity of the paths stated in Theorem 2.3.

4.1. Two-sided sticky Brownian motion. We here introduce the two-sided sticky Brownian motion on the interval $[0,1]$. Although its construction is analogous to the one of the sticky Brownian motion on $[0,+\infty)$, see e.g. Varadhan (2007), we outline the general strategy and provide the details that are relevant for our purposes.

We start by introducing the Skorokhod problem on the interval $[0,1]$. Given a function $u \in C[0,+\infty)$ such that $u(0) \in[0,1]$ a triple of continuous functions 
$\left(v, a_{0}, a_{1}\right)$, where $0 \leq v \leq 1$ and $a_{0}, a_{1}$ are increasing solves the Skorokhod problem on the interval $[0,1]$ if and only if $v=u+a_{0}-a_{1}, a_{0}(0)=a_{1}(0)=0$, and the measure $d a_{i}$ is carried on the set $\{t: v(t)=i\}, i=0,1$.

As shown in Kruk et al. (2007) there exists a unique solution to this problem given by $v=\Gamma(u), a_{i}=A_{i}(u)$ where the maps $\Gamma$ and $A_{i}$ are explicitly constructed, $i=$ 1,0 . Moreover, these maps are continuous in the uniform topology and progressively measurable namely, the values of $v$ and $a_{i}$ at time $t$ depend only on the values of $u$ at the times $[0, t]$.

The Brownian motion on the interval $[0,1]$ with elastic reflection at the endpoints and initial condition $y \in[0,1]$ can then be defined as $Y=\Gamma(B)$ where $B$ is a standard Brownian motion on $\mathbf{R}$ starting from $y$.

Given $\theta=\left(\theta_{0}, \theta_{1}\right) \in(0,+\infty)^{2}$, following Varadhan (2007) we define the continuous strictly increasing process

$$
\sigma(t)=t+\frac{1}{\theta_{0}} a_{0}(t)+\frac{1}{\theta_{1}} a_{1}(t), \quad t \geq 0
$$

and denote by $\sigma^{-1}$ its inverse. The two sided $\theta$-sticky Brownian motion on $[0,1]$ with initial condition $x \in[0,1]$ is then defined by $X(t)=Y\left(\sigma^{-1}(t)\right), t \geq 0$, where $Y$ is the Brownian motion on the interval $[0,1]$ with elastic reflection on the endpoints and initial condition $x$.

Arguing as in Varadhan (2007), an application of Itô's formula shows that for each $f \in C^{2}[0,1]$

$$
\begin{aligned}
& M^{f}(t):=f(X(t))-f(X(0)) \\
& \quad-\int_{0}^{t} d s\left[\frac{1}{2} f^{\prime \prime}(X(s)) \mathbf{1}_{(0,1)}(X(s))+\theta_{0} f^{\prime}(0) \mathbf{1}_{\{0\}}(X(s))-\theta_{1} f^{\prime}(1) \mathbf{1}_{\{1\}}(X(s))\right]
\end{aligned}
$$

is a continuous martingale with quadratic variation

$$
\left[M^{f}\right](t)=\int_{0}^{t} d s f^{\prime}(X(s))^{2} \mathbf{1}_{(0,1)}(X(s))
$$

By using (4.1), routine manipulations yield that $X$ is a Feller process on the state space $[0,1]$ with generator $\mathcal{L}$ given by $\mathcal{L} f=f^{\prime \prime} / 2$ on the domain

$$
\mathcal{D}(\mathcal{L})=\left\{f \in C^{2}[0,1]: 2 \theta_{0} f^{\prime}(0)=f^{\prime \prime}(0), 2 \theta_{1} f^{\prime}(1)=-f^{\prime \prime}(1)\right\} .
$$

We denote by $\mathbf{P}_{x}^{\theta}$ the law of a two-sided $\theta$-sticky Brownian motion started at $x \in$ $[0,1]$. Observe that in the limit $\theta \rightarrow 0$ this process converges to the Brownian motion in $[0,1]$ with absorption at the endpoints, so that the notation is consistent with Section 2.

The resolvent equation for $\mathcal{L}$ has the form of a Sturm-Liouville problem on the interval $[0,1]$ so that it is possible to obtain an explicit expression for the resolvent kernel $r_{\lambda}$,

$$
r_{\lambda}(x, d y)= \begin{cases}g_{\lambda}(x, y) d y+g_{\lambda}^{0}(x) \delta_{1}(d y) & x \leq y \\ g_{\lambda}(y, x) d y+g_{\lambda}^{1}(x) \delta_{0}(d y) & x>y\end{cases}
$$


where

$$
\begin{aligned}
g_{\lambda}(x, y) & =2 W^{-1}\left(2 \theta_{0} \operatorname{ch}(\sqrt{2 \lambda} x)+\sqrt{2 \lambda} \operatorname{sh}(\sqrt{2 \lambda} x)\right) \\
& \times\left(2 \theta_{1} \operatorname{ch}(\sqrt{2 \lambda}(1-y))+\sqrt{2 \lambda} \operatorname{sh}(\sqrt{2 \lambda}(1-y))\right) \\
g_{\lambda}^{0}(x) & =2 W^{-1}\left(2 \theta_{0} \operatorname{ch}(\sqrt{2 \lambda} x)+\sqrt{2 \lambda} \operatorname{sh}(\sqrt{2 \lambda} x)\right) \\
g_{\lambda}^{1}(x) & =2 W^{-1}\left(2 \theta_{1} \operatorname{ch}(\sqrt{2 \lambda}(1-x))+\sqrt{2 \lambda} \operatorname{sh}(\sqrt{2 \lambda}(1-x))\right)
\end{aligned}
$$

in which

$$
W=4 \lambda\left(\theta_{0}+\theta_{1}\right) \operatorname{ch} \sqrt{2 \lambda}+2 \sqrt{2 \lambda}\left(2 \theta_{0} \theta_{1}+\lambda\right) \operatorname{sh} \sqrt{2 \lambda} .
$$

According to (4.3), the transition function of a two-sided $\theta$-sticky Brownian motion can be written as

$$
p_{t}(x, d y)=q_{t}(x, y) d y+q_{t}^{1}(x) \delta_{0}(d y)+q_{t}^{0}(x) \delta_{1}(d y)
$$

where the Laplace transform of $q_{t}, q_{t}^{0}$, and $q_{t}^{1}$ are $g_{\lambda}, g_{\lambda}^{0}$, and $g_{\lambda}^{1}$, respectively.

We conclude this subsection with two technical lemmata.

Lemma 4.1. Given $T>0$ there exists $C>0$ such that for any $t \in[0, T]$ and $\theta \in(0,+\infty)^{2}$

$$
\begin{gathered}
\sup _{y \in(0,1)}\left|q_{t}(0, y)-2 \theta_{0} \mathbf{P}_{y}^{0}\left(\tau_{0} \leq t\right)\right| \leq C\|\theta\|^{2} \\
\sup _{y \in(0,1)}\left|q_{t}(1, y)-2 \theta_{1} \mathbf{P}_{y}^{0}\left(\tau_{1} \leq t\right)\right| \leq C\|\theta\|^{2} .
\end{gathered}
$$

Moreover, for $b \in\{0,1\}$,

$$
1-p_{t}(b,\{b\}) \leq C\|\theta\| \quad p_{t}(b,\{1-b\}) \leq C\|\theta\| .
$$

Proof: We start by proving the first bound in (4.4). By an explicit computation

$$
\begin{aligned}
& \left.\frac{\partial}{\partial \theta_{0}} g_{\lambda}(0, y)\right|_{\theta=0}=\frac{2 \operatorname{sh}(\sqrt{2 \lambda}(1-y))}{\lambda \operatorname{sh} \sqrt{2 \lambda}} \\
& \left.\frac{\partial}{\partial \theta_{1}} g_{\lambda}(0, y)\right|_{\theta=0}=0 .
\end{aligned}
$$

We now claim that there exists a constant $C$ such that for any $\lambda \in \mathbf{C}$ with $\Re(\lambda)=1$ and $\theta \in(0,+\infty)^{2}$ :

$$
\sup _{y \in(0,1)}\left|g_{\lambda}(0, y)-\theta_{0} \frac{\partial}{\partial \theta_{0}} g_{\lambda}(0, y)\right|_{\theta=0} \mid \leq \frac{C\|\theta\|^{2}}{|\lambda|^{3 / 2}} .
$$

By Eq. 2.2.4 (1) of Borodin and Salminen (2002), $\operatorname{sh}(\sqrt{2 \lambda}(1-y)) /(\lambda \operatorname{sh} \sqrt{2 \lambda})$ is the Laplace transform of $P_{y}^{0}\left(\tau_{0} \leq t\right)$. The statement follows.

To prove (4.6), by an explicit computation, recalling (4.3),

$$
\begin{aligned}
& g_{\lambda}(0,1-y)-\left.\theta_{0} \frac{\partial}{\partial \theta_{0}} g_{\lambda}(0,1-y)\right|_{\theta=0} \\
& =\frac{4 \theta_{0}\left[\theta_{1} \sqrt{\lambda} \operatorname{ch}(y \sqrt{2 \lambda})-\left(\sqrt{2} \theta_{0} \theta_{1}+\left(\theta_{0}+\theta_{1}\right) \sqrt{\lambda} \operatorname{cth}(\sqrt{2 \lambda})\right) \operatorname{sh}(y \sqrt{2 \lambda})\right]}{\lambda^{3 / 2} \operatorname{sh}(\sqrt{2 \lambda})\left[\sqrt{2}\left(2 \theta_{0} \theta_{1}+\sqrt{\lambda}\right)+2\left(\theta_{0}+\theta_{1}\right) \operatorname{cth}(\sqrt{2 \lambda})\right]} .
\end{aligned}
$$


By using that for $\Re(\lambda)=1$ we have $\sqrt{2 \lambda}=\sqrt{|\lambda|+1}+i \operatorname{sgn}(\Im(\lambda)) \sqrt{|\lambda|-1}$, $|\operatorname{cth}(\sqrt{2 \lambda})|$ is bounded, and

$$
\sup _{y \in(0,1)}\left|\frac{\operatorname{ch}(y \sqrt{2 \lambda})}{\operatorname{sh}(\sqrt{2 \lambda})}\right|+\sup _{y \in(0,1)}\left|\frac{\operatorname{sh}(y \sqrt{2 \lambda})}{\operatorname{sh}(\sqrt{2 \lambda})}\right| \leq C,
$$

for some $C>0$ independent of $\lambda$, a straightforward computation yields (4.6).

The second bound in (4.4) is obtained by symmetry. To prove (4.5) it is enough to show that there exists a constant $C$ such that for any $\lambda \in \mathbf{C}$ with $\Re(\lambda)=1$ and $\theta \in(0,+\infty)^{2}:$

$$
\left|g_{\lambda}^{0}(1)-\frac{1}{\lambda}\right| \leq \frac{C\|\theta\|}{|\lambda|^{3 / 2}}, \quad \quad\left|g_{\lambda}^{0}(0)\right| \leq \frac{C\|\theta\|}{|\lambda|^{3 / 2}} .
$$

This follows by direct computations.

Lemma 4.2. Given $T>0$ and $\psi \in C_{0}(0,1)$ or $\psi=\mathbf{1}_{(0,1)}$ there exists $C>0$ such that for any $t \in[0, T]$ and $\theta \in(0,+\infty)^{2}$

$$
\sup _{x \in(0,1)}\left|\mathbf{E}_{x}^{\theta}[\psi(X(t))]-\mathbf{E}_{x}^{0}[\psi(X(t))]\right| \leq C\|\theta\| .
$$

Proof: Since $\psi(0)=\psi(1)=0$ and the two-sided $\theta$-sticky Brownian motion coincides with the Brownian motion with absorption at the boundary until the processes reaches the boundary,

$$
\mathbf{E}_{x}^{\theta}[\psi(X(t))]-\mathbf{E}_{x}^{0}[\psi(X(t))]=\mathbf{E}_{x}^{\theta}[\psi(X(t)), \tau \leq t] .
$$

By the strong Markov property and Lemma 4.1,

$$
\begin{gathered}
\left|\mathbf{E}_{x}^{\theta}[\psi(X(t)), \tau \leq t]\right| \leq \int_{0}^{t} \mathbf{P}_{x}^{\theta}(\tau \in d s, X(\tau)=0) \mathbf{E}_{0}^{\theta}[|\psi(X(t-s))|] \\
\quad+\int_{0}^{t} \mathbf{P}_{x}^{\theta}(\tau \in d s, X(\tau)=1) \mathbf{E}_{1}^{\theta}[|\psi(X(t-s))|] \leq C\|\psi\|_{\infty}\|\theta\|,
\end{gathered}
$$

which completes the proof.

4.2. Convergence to the boundary driven Brownian gas. Given $n \in \mathbf{N}$, we introduce the empirical measure as the map $\pi_{n}:[0,1]^{n} \rightarrow \Omega$ defined by

$$
\pi_{n}\left(x_{1}, \ldots, x_{n}\right):=\sum_{k=1}^{n} \delta_{x_{k}},
$$

where we understand that if $x_{k} \in\{0,1\}$ then it gives no weight to the right hand side. For $T>0$, with a slight abuse of notation, we denote by $\pi_{n}$ also the map from $C\left([0, T] ;[0,1]^{n}\right)$ to $C([0, T] ; \Omega)$ defined by $\pi_{n}\left(x_{1}, \ldots, x_{n}\right)(t):=\pi_{n}\left(x_{1}(t), \ldots, x_{n}(t)\right)$, $t \in[0, T]$.

We are going to consider the empirical measure of $n$ independent sticky Brownian motions and consider the limit $n \rightarrow \infty$ when the stickiness parameter $\theta=\theta^{n}$ vanishes in such a way that $n \theta^{n}=\left(n \theta_{0}^{n}, n \theta_{1}^{n}\right) \rightarrow\left(\lambda_{0}, \lambda_{1}\right)=\lambda$. In order to obtain the convergence to the boundary driven Brownian gas the initial datum has to be suitably prepared. Let $\omega \in \Omega$ be the initial datum for the boundary driven Brownian gas and consider first the case in which $\omega$ has finite mass so that $\omega=\sum_{k=1}^{A} \delta_{x_{k}}$ for some $A \in \mathbf{N}$ and $x_{1}, \ldots, x_{A} \in(0,1)$. Then, the initial datum for the $n$ independent sticky Brownian motions is chosen as follows. The first $A$ particles start at the 
points $x_{1}, \ldots, x_{A}$, half of the remaining $n-A$ start at 0 , and the other half at 1 . In this situation, as the stickiness parameter is of order $1 / n$, the particles initially in $(0,1)$ will essentially perform a Brownian motion with absorption at the endpoints. On the other hand, again by the scaling of the stickiness parameter, out of the approximately $n / 2$ particles initially at the end-point $\{0\}$ essentially only a finite number will be able to move inside $(0,1)$ by a strictly positive distance independent of $n$. Together with the analogous mechanism at the end-point $\{1\}$, this will produce the effect of the boundary reservoirs in the limiting process.

We now describe how the initial state is prepared in the general situation in which the mass of $\omega$ is possibly unbounded. Given $\omega \in \Omega$ and $n \in \mathbf{N}$ let $x^{n}=$ $x^{n}(\omega)=\left(x_{1}^{n}, \ldots, x_{n}^{n}\right) \in[0,1]^{n}$ be the following triangular array. Choose a sequence $a_{n} \downarrow 0$ such that $A_{n}:=\omega\left(a_{n}, 1-a_{n}\right)=o(n)$. Define $x_{k}^{n}, k=1, \ldots, A_{n}$ so that $\omega^{n}:=\omega\left(\cdot \cap\left(a_{n}, 1-a_{n}\right)\right)=\sum_{k=1}^{A_{n}} \delta_{x_{k}^{n}}$. Define also $x_{k}^{n}=0, k=A_{n}+1, \ldots, A_{n}+$ $\left\lfloor\left(n-A_{n}\right) / 2\right\rfloor$, and $x_{k}^{n}=1, k=A_{n}+\left\lfloor\left(n-A_{n}\right) / 2\right\rfloor+1, \ldots, n$. Observe that, as simple to check, $\omega^{n} \rightarrow \omega$ in $\Omega$.

The Poissonian limit of independent sticky Brownian motions is then stated in the next theorem in which $\Omega$ is endowed with the topology introduced in Section 2 and $C([0, T] ; \Omega)$ with the corresponding uniform topology.

Theorem 4.3. Given $\omega \in \Omega$ and $n \in \mathbf{N}$, set $\mathbb{P}_{\omega}^{n}:=\left(\prod_{k=1}^{n} \mathbf{P}_{x_{k}^{n}}^{\theta^{n}}\right) \circ \pi_{n}^{-1}$ where the initial data $x^{n}=x^{n}(\omega)$ are as above and $\theta^{n}=\left(\lambda_{0} / n, \lambda_{1} / n\right), \lambda_{0}, \lambda_{1}>0$. For each $T>0$ the sequence $\left\{\mathbb{P}_{\omega}^{n}\right\}$, as probabilities on $C([0, T] ; \Omega)$, converges weakly to $\mathbb{P}_{\omega}$.

The proof of this theorem is accomplished by first proving tightness of $\left\{\mathbb{P}_{\omega}^{n}\right\}$, then identifying its cluster points by showing that their finite dimensional distributions are Markovian with transition function $P_{t}$.

4.3. Tightness. Since $\Omega$ is not Polish, there are few technicalities in the proof of the tightness. We shall apply the compact containment criterion discussed in Jakubowski (1986). In order to apply Theorem 3.1 there, we observe that the family of functions $\Omega \ni \omega \mapsto \omega(\psi) \in \mathbf{R}, \psi \in C_{K}^{2}(0,1)$, separates the points in $\Omega$ and it is closed under addition. The tightness of the sequence $\left\{\mathbb{P}_{\omega}^{n}\right\}$ is thus achieved once we show that the following two conditions are met:

(i) there exists a sequence of compacts $K_{\ell} \subset \subset \Omega$ such that

$$
\lim _{\ell \rightarrow \infty} \sup _{n} \mathbb{P}_{\omega}^{n}\left(\exists t \in[0, T]: \omega(t) \notin K_{\ell}\right)=0 ;
$$

(ii) for each $\psi \in C_{K}^{2}(0,1)$ the sequence $\left\{\mathbb{P}_{\omega}^{n} \circ \chi_{\psi}^{-1}\right\}$ is tight on $C([0, T])$, where $\chi_{\psi}: C([0, T] ; \Omega) \ni \omega \mapsto \omega(\psi) \in C([0, T])$.

Recalling Lemma A.1, the following statement implies that condition (i) holds.

Lemma 4.4.

$$
\lim _{\ell \rightarrow+\infty} \sup _{n} \mathbb{P}_{\omega}^{n}\left(\sup _{t \in[0, T]} \omega(t)(m)>\ell\right)=0 .
$$

Proof: Let $X$ be a two-sided sticky Brownian motion with parameter $\theta=\left(\theta_{0}, \theta_{1}\right)$ and initial datum $x$. By (4.1)

$$
\begin{aligned}
& m(X(t))=m(x) \\
& \quad+\int_{0}^{t} d s\left[-\mathbf{1}_{(0,1)}(X(s))+\theta_{0} \mathbf{1}_{\{0\}}(X(s))+\theta_{1} \mathbf{1}_{\{1\}}(X(s))\right]+M(t),
\end{aligned}
$$


where $M$ is a continuous square integrable martingale with quadratic variation

$$
[M](t)=\int_{0}^{t} d s m^{\prime}(X(s))^{2} \mathbf{1}_{(0,1)}(X(s))
$$

By considering $n$ independent sticky Brownian motions, from (4.8) we deduce

$$
\omega(t)(m) \leq \omega(0)(m)+2 n\left\|\theta^{n}\right\| t+N(t), \quad \mathbb{P}_{\omega}^{n} \text {-a.s. }
$$

where $N$ is a $\mathbb{P}_{\omega}^{n}$ continuous square integrable martingale with quadratic variation

$$
[N](t)=\int_{0}^{t} d s \omega(s)\left(\left(m^{\prime}\right)^{2} \mathbf{1}_{(0,1)}\right)
$$

By assumption, $\mathbb{P}_{\omega}^{n}$-a.s., $\omega(0)(m)=\pi_{n}\left(x^{n}\right)(m)$, which is uniformly bounded. Since $n\left\|\theta^{n}\right\|=\|\lambda\|$, it is therefore enough to show that

$$
\lim _{\ell \rightarrow+\infty} \sup _{n} \mathbb{P}_{\omega}^{n}\left(\sup _{t \in[0, T]} N(t)>\ell\right)=0 .
$$

By Doob's inequality

$$
\begin{aligned}
& \mathbb{P}_{\omega}^{n}\left(\sup _{t \in[0, T]} N(t)>\ell\right) \leq \frac{1}{\ell^{2}} \mathbb{E}_{\omega}^{n}([N](T)) \\
& \leq \frac{1}{\ell^{2}} \int_{0}^{T} d t \mathbb{E}_{\omega}^{n}[|\omega(t)|]=\frac{1}{\ell^{2}} \sum_{k=1}^{n} \int_{0}^{T} d t \mathbf{P}_{x_{k}^{n}}^{\theta^{n}}(X(t) \in(0,1)) .
\end{aligned}
$$

In view of Lemma 4.2 and recalling that $\tau=\inf \{t \geq 0: X(t) \in\{0,1\}\}$,

$$
\begin{aligned}
& \int_{0}^{T} d t \mathbf{P}_{x}^{\theta}(X(t) \in(0,1)) \leq \int_{0}^{T} d t\left[\mathbf{P}_{x}^{0}(X(t) \in(0,1))+C\|\theta\|\right] \\
& \quad \leq \int_{0}^{+\infty} d t \mathbf{P}_{x}^{0}(\tau>t)+C T\|\theta\|=\mathbf{E}_{x}^{0}(\tau)+C T\|\theta\|=m(x)+C T\|\theta\|
\end{aligned}
$$

By plugging this bound into (4.11), the estimate (4.10) follows.

By standard tightness criterion on $C([0, T])$, the following equicontinuity yields condition (ii).

Lemma 4.5. For each $\psi \in C_{K}^{2}(0,1)$ and $\varepsilon>0$

$$
\lim _{\delta \downarrow 0} \sup _{n} \mathbb{P}_{\omega}^{n}\left(\sup _{|t-s|<\delta}|\omega(t)(\psi)-\omega(s)(\psi)|>\varepsilon\right)=0 .
$$

Proof: By a simple inclusion of events, see Billingsley (1999) Thm. 8.3, it is enough to show that for each $\varepsilon>0$

$$
\lim _{\delta \downarrow 0} \sup _{n} \sup _{s \in[0, T-\delta]} \frac{1}{\delta} \mathbb{P}_{\omega}^{n}\left(\sup _{t \in[s, s+\delta]}|\omega(t)(\psi)-\omega(s)(\psi)|>\varepsilon\right)=0 .
$$

Fix $s \in[0, T-\delta]$ and let $X$ be a two-sided $\theta$-sticky Brownian motion. By (4.1)

$$
\psi(X(t))-\psi(X(s))=\frac{1}{2} \int_{s}^{t} d u \psi^{\prime \prime}(X(u))+M^{s}(t)
$$

where $M^{s}(t), t \in[s, T]$ is a continuous square integrable martingale with quadratic variation

$$
\left[M^{s}\right](t)=\int_{s}^{t} d u \psi^{\prime}(X(u))^{2}
$$


By considering $n$ independent sticky Brownian motions, from (4.13) we deduce, $\mathbb{P}_{\omega}^{n}$-a.s.

$$
\sup _{t \in[s, s+\delta]}|\omega(t)(\psi)-\omega(s)(\psi)| \leq \frac{1}{2} \int_{s}^{s+\delta} d u\left|\omega(u)\left(\psi^{\prime \prime}\right)\right|+\sup _{t \in[s, s+\delta]}\left|N^{s}(t)\right|
$$

where $N^{s}(t), t \in[s, T]$ is a $\mathbb{P}_{\omega}^{n}$ continuous square integrable martingale with quadratic variation

$$
\left[N^{s}\right](t)=\int_{s}^{t} d u \omega(u)\left(\left(\psi^{\prime}\right)^{2}\right)
$$

Let $K:=\operatorname{supp} \psi$. To control the bounded variation term on the right hand side of (4.14) we apply Chebyshev's and Cauchy-Schwarz's inequalities to deduce

$$
\mathbb{P}_{\omega}^{n}\left(\int_{s}^{s+\delta} d u\left|\omega(u)\left(\psi^{\prime \prime}\right)\right|>\varepsilon\right) \leq \frac{\delta}{\varepsilon^{2}}\left\|\psi^{\prime \prime}\right\|_{\infty}^{2} \mathbb{E}_{\omega}^{n} \int_{s}^{s+\delta} d u[\omega(u)(K)]^{2} .
$$

We claim that

$$
\sup _{n} \sup _{t \in[0, T]} \mathbb{E}_{\omega}^{n}\left[\omega(t)(K)^{2}\right]<+\infty .
$$

Together with the previous bound this yields

$$
\lim _{\delta \downarrow 0} \sup _{n} \sup _{s \in[0, T-\delta]} \frac{1}{\delta} \mathbb{P}_{\omega}^{n}\left(\int_{s}^{s+\delta} d u\left|\omega(u)\left(\psi^{\prime \prime}\right)\right|>\varepsilon\right)=0 .
$$

To control the martingale part on the right hand side of (4.14), we apply the BDG inequality (see e.g. Thm. IV.4.1 of Revuz and Yor, 1991) and Cauchy-Schwarz inequality to deduce

$$
\begin{gathered}
\mathbb{P}_{\omega}^{n}\left(\sup _{t \in[s, s+\delta]}\left|N^{s}(t)\right|>\varepsilon\right) \leq C \mathbb{E}_{\omega}^{n}\left(\left[N^{s}\right](s+\delta)^{2}\right) \\
\leq C \delta\left\|\psi^{\prime}\right\|_{\infty}^{4} \int_{s}^{s+\delta} d u \mathbb{E}_{\omega}^{n}\left(\omega(u)(K)^{2}\right)
\end{gathered}
$$

By using (4.15) we thus get

$$
\lim _{\delta \downarrow 0} \sup _{n} \sup _{s \in[0, T-\delta]} \frac{1}{\delta} \mathbb{P}_{\omega}^{n}\left(\sup _{t \in[s, s+\delta]}\left|N^{s}(t)\right|>\varepsilon\right)=0 .
$$

It remains to prove the claim (4.15). By a simple computation

$$
\mathbb{E}_{\omega}^{n}\left(\omega(t)(K)^{2}\right) \leq\left\{\mathbb{E}_{\omega}^{n}(\omega(t)(K))\right\}^{2}+\mathbb{E}_{\omega}^{n}(\omega(t)(K))
$$

We conclude by observing that for some constant $C>0$ we have $\mathbf{1}_{K} \leq C m$ and taking the expectation of (4.9).

4.4. Identification of the limit. We here conclude the proof of Theorem 4.3 by identifying the finite dimensional distributions, via their characteristic function (cfr. Lemma A.3), of the cluster points of the sequence $\left\{\mathbb{P}_{\omega}^{n}\right\}$. Recall that $P_{t}, t \geq 0$ is the time homogeneous transition function defined in (2.2).

Proposition 4.6. For each $r \in \mathbf{N}, 0 \leq t_{1}<\cdots<t_{r} \leq T$, and $\psi_{1}, \ldots, \psi_{r} \in$ $C_{K}(0,1)$

$$
\lim _{n \rightarrow+\infty} \mathbb{E}_{\omega}^{n}\left(e^{i \sum_{h=1}^{r} \omega\left(t_{h}\right)\left(\psi_{h}\right)}\right)=\int \ldots \int \prod_{h=1}^{r} P_{t_{h}-t_{h-1}}\left(\eta_{h-1}, d \eta_{h}\right) e^{i \eta_{h}\left(\psi_{h}\right)},
$$

where we understand that $t_{0}=0$ and $\eta_{0}=\omega$. 
We start with the case $r=1$.

Lemma 4.7. For each $t \in[0, T]$ and $\psi \in C_{0}(0,1)$

$$
\lim _{n \rightarrow+\infty} \mathbb{E}_{\omega}^{n}\left[e^{i \omega(t)(\psi)}\right]=\int P_{t}(\omega, d \eta) e^{i \eta(\psi)} .
$$

Proof: For $t=0$ the statement follows directly from the construction of the triangular array $x^{n}$. For $t>0$ we write

$$
\begin{aligned}
\prod_{k=1}^{n} \mathbf{E}_{x_{k}^{n}}^{\theta^{n}}\left[e^{i \psi(X(t))}\right]= & \left(\mathbf{E}_{0}^{\theta^{n}}\left[e^{i \psi(X(t))}\right]\right)^{\left\lfloor\frac{n-A_{n}}{2}\right\rfloor}\left(\mathbf{E}_{1}^{\theta^{n}}\left[e^{i \psi(X(t))}\right]\right)^{\left\lceil\frac{n-A_{n}}{2}\right\rceil} \\
& \times \prod_{k=1}^{A_{n}} \mathbf{E}_{x_{k}^{n}}^{\theta^{n}}\left[e^{i \psi(X(t))}\right] .
\end{aligned}
$$

Since $A_{n}=o(n)$ we have $n^{-1}\left\lfloor\left(n-A_{n}\right) / 2\right\rfloor \rightarrow 1 / 2$. Hence, by applying Lemma 4.1,

$$
\begin{aligned}
\lim _{n \rightarrow+\infty}\left(\mathbf{E}_{0}^{\theta^{n}}\left[e^{i \psi(X(t))}\right]\right)^{\left\lfloor\frac{n-A_{n}}{2}\right\rfloor} & =\lim _{n \rightarrow+\infty}\left(1+\mathbf{E}_{0}^{\theta^{n}}\left[e^{i \psi(X(t))}-1\right]\right)^{\left\lfloor\frac{n-A_{n}}{2}\right\rfloor} \\
& =\exp \left\{\lambda_{0} \int_{0}^{1} d x \mathbf{P}_{x}^{0}\left(\tau_{0} \leq t\right)\left(e^{i \psi(x)}-1\right)\right\} .
\end{aligned}
$$

For the same reasons,

$$
\lim _{n \rightarrow+\infty}\left(\mathbf{E}_{1}^{\theta^{n}}\left[e^{i \psi(X(t))}\right]\right)^{\left\lceil\frac{n-A_{n}}{2}\right\rceil}=\exp \left\{\lambda_{1} \int_{0}^{1} d x \mathbf{P}_{x}^{0}\left(\tau_{1} \leq t\right)\left(e^{i \psi(x)}-1\right)\right\} .
$$

Recalling (2.2), to complete the proof it remains to show

$$
\lim _{n \rightarrow+\infty} \prod_{k=1}^{A_{n}} \mathbf{E}_{x_{k}^{n}}^{\theta^{n}}\left[e^{i \psi(X(t))}\right]=\prod_{x \in \omega} \mathbf{E}_{x}^{0}\left[e^{i \psi(X(t))}\right] .
$$

In order to prove (4.19), we set

$$
R_{k}^{n}:=\mathbf{E}_{x_{k}^{n}}^{\theta^{n}}\left[e^{i \psi(X(t))}\right]-\mathbf{E}_{x_{k}^{n}}^{0}\left[e^{i \psi(X(t))}\right]=\mathbf{E}_{x_{k}^{n}}^{\theta^{n}}\left[e^{i \psi(X(t))}-1\right]-\mathbf{E}_{x_{k}^{n}}^{0}\left[e^{i \psi(X(t))}-1\right]
$$

and observe, as follows from Lemma 4.2, that for each $t>0$ there exists a constant $C$ independent of $n$ and $k$ such that $\left|R_{k}^{n}\right| \leq C / n$. Since $A_{n}=o(n)$ and $\omega^{n}=\sum_{k=1}^{A_{n}} \delta_{x_{k}^{n}}$, it is therefore enough to show

$$
\lim _{n \rightarrow+\infty} \prod_{x \in \omega^{n}} \mathbf{E}_{x}^{0}\left[e^{i \psi(X(t))}\right]=\prod_{x \in \omega} \mathbf{E}_{x}^{0}\left[e^{i \psi(X(t))}\right],
$$

which is implied by

$$
\sum_{x \in \omega}\left|\mathbf{E}_{x}^{0}\left[e^{i \psi(X(t))}-1\right]\right| \leq \sum_{x \in \omega} \mathbf{P}_{x}^{0}(\tau>t) \leq \frac{1}{t} \sum_{x \in \omega} \mathbf{E}_{x}^{0}(\tau)=\frac{1}{t} \omega(m)<+\infty .
$$

Remark 4.8. The argument in the above proof actually implies the following uniform statement that will be used in the sequel. Let $\ell>0, \psi \in C_{0}(0,1)$, and $\varepsilon_{n} \downarrow 0$. Then

$$
\lim _{n \rightarrow+\infty} \sup _{x \in B_{\ell}^{n}}\left|\prod_{k=1}^{n} \mathbf{E}_{x_{k}}^{\theta^{n}}\left[e^{i \psi(X(t))}\right]-\int P_{t}\left(\sum_{k=1}^{n} \delta_{x_{k}}, d \eta\right) e^{i \eta(\psi)}\right|=0,
$$


where

$$
\begin{aligned}
& B_{\ell}^{n}=\left\{x \in[0,1]^{n}:\left|\left\{k: x_{k} \in(0,1)\right\}\right| \leq \ell\right. \\
&\left.\frac{1}{n}||\left\{k: x_{k}=0\right\}\left|-\frac{n}{2}\right| \leq \varepsilon_{n}, \frac{1}{n}||\left\{k: x_{k}=1\right\}\left|-\frac{n}{2}\right| \leq \varepsilon_{n}\right\} .
\end{aligned}
$$

As usual, we understand that if $x_{k} \in\{0,1\}$ then it gives no weight to the sum on the second term in (4.20).

The proof of Proposition 4.6 is achieved by induction on $r$. The recursive step is the content of the next lemma.

Lemma 4.9. Assume that the conclusion of Proposition 4.6 holds for some $r \in \mathbf{N}$ then it holds for $r+1$.

Proof: The proof is essentially follows from the Markov property of the sticky Brownian motions and Lemma 4.7. Since the transition function $P_{t}$ is not Feller, there is however a continuity issue that will be handled by a suitable approximation.

We first show that, with probability close to one for $n$ large, the configuration of the sticky Brownian motions at some positive time meets the conditions on the initial datum in Remark 4.8. More precisely, letting $\mathcal{P}^{n}:=\prod_{k=1}^{n} \mathbf{P}_{x_{k}^{n}}^{\theta^{n}}$ be the law of the $n$ independent sticky Brownian motions, we shall prove the two following bounds.

(i) For each $t \in(0, T]$

$$
\lim _{\ell \rightarrow+\infty} \sup _{n} \mathcal{P}^{n}\left(\sum_{k=1}^{n} \delta_{X_{k}(t)} \notin B_{\ell}^{1}\right)=0, \quad B_{\ell}^{1}:=\{\omega \in \Omega:|\omega| \leq \ell\} .
$$

(ii) There exists a sequence $\varepsilon_{n} \rightarrow 0$ such that for each $t \in[0, T]$

$$
\lim _{n \rightarrow+\infty} \mathcal{P}^{n}\left(X(t) \notin B_{\varepsilon_{n}}^{2}\right)=0,
$$

where

$$
B_{\varepsilon}^{2}:=\left\{x \in[0,1]^{n}: \frac{1}{n}||\left\{k: x_{k}=0\right\}\left|-\frac{n}{2}\right| \leq \varepsilon, \frac{1}{n}||\left\{k: x_{k}=1\right\}\left|-\frac{n}{2}\right| \leq \varepsilon\right\} .
$$

To prove (i) we observe that by Lemma 4.2 there exists a constant $C>0$ such that

$$
\begin{aligned}
\int d \mathcal{P}^{n} & \left|\left\{k: X_{k}(t) \in(0,1)\right\}\right|=\sum_{k=1}^{n} \mathbf{P}_{x_{k}^{n}}^{\theta^{n}}(X(t) \in(0,1)) \\
& =\sum_{k=1}^{n} \mathbf{P}_{x_{k}^{n}}^{0}(X(t) \in(0,1))+C=\sum_{k=1}^{n} \mathbf{P}_{x_{k}^{n}}^{0}(\tau>t)+C \\
& \leq \frac{1}{t} \sum_{k=1}^{n} \mathbf{E}_{x_{k}^{n}}^{0}(\tau)+C=\frac{1}{t} \sum_{k=1}^{n} m\left(x_{k}^{n}\right)+C,
\end{aligned}
$$

which is uniformly bounded in $n$. We conclude by Chebyshev's inequality.

To prove (ii), it is enough to show that

$$
\lim _{n \rightarrow+\infty} \mathcal{P}^{n}\left(\left|\sum_{k=1}^{n} \mathbf{1}_{\{b\}}\left(X_{k}(t)\right)-\frac{n}{2}\right| \geq n \varepsilon_{n}\right)=0, \quad b \in\{0,1\} .
$$


We consider only the case $b=0$ and write

$$
\begin{aligned}
& \left|\sum_{k=1}^{n} \mathbf{1}_{\{0\}}\left(X_{k}(t)\right)-\frac{n}{2}\right| \\
& \leq\left|\sum_{k=1}^{A_{n}} \mathbf{1}_{\{0\}}\left(X_{k}(t)\right)\right|+\left|\sum_{k=A_{n}+1}^{A_{n}+\left\lfloor\left(n-A_{n}\right) / 2\right\rfloor}\left(\mathbf{1}_{\{0\}}\left(X_{k}(t)\right)-1\right)\right|+\left|\left\lfloor\frac{n-A_{n}}{2}\right\rfloor-\frac{n}{2}\right| \\
& +\left|\sum_{k=A_{n}+\left\lfloor\left(n-A_{n}\right) / 2\right\rfloor+1}^{n} \mathbf{1}_{\{0\}}\left(X_{k}(t)\right)\right| \\
& \leq A_{n}+\left\lceil\frac{A_{n}}{2}\right\rceil+\left|\sum_{k=A_{n}+1}^{A_{n}+\left\lfloor\left(n-A_{n}\right) / 2\right\rfloor}\left(\mathbf{1}_{\{0\}}\left(X_{k}(t)\right)-1\right)\right|+\left|\sum_{k=A_{n}+\left\lfloor\left(n-A_{n}\right) / 2\right\rfloor+1}^{n} \mathbf{1}_{\{0\}}\left(X_{k}(t)\right)\right| .
\end{aligned}
$$

By setting $\varepsilon_{n}=9\left(A_{n}+1\right) /(2 n)$, since $A_{n}+\left\lceil A_{n} / 2\right\rceil<n \varepsilon_{n} / 3$, it is enough to show that

$$
\begin{aligned}
& \lim _{n \rightarrow+\infty} \mathcal{P}^{n}\left(\left|\sum_{k=A_{n}+1}^{A_{n}+\left\lfloor\left(n-A_{n}\right) / 2\right\rfloor}\left(\mathbf{1}_{\{0\}}\left(X_{k}(t)\right)-1\right)\right| \geq \frac{n \varepsilon_{n}}{3}\right)=0 \\
& \lim _{n \rightarrow+\infty} \mathcal{P}^{n}\left(\left|\sum_{k=A_{n}+\left\lfloor\left(n-A_{n}\right) / 2\right\rfloor+1}^{n} \mathbf{1}_{\{0\}}\left(X_{k}(t)\right)\right| \geq \frac{n \varepsilon_{n}}{3}\right)=0 .
\end{aligned}
$$

Since $x_{k}^{n}=0$ for $k=A_{n}+1, \ldots, A_{n}+\left\lfloor\left(n-A_{n}\right) / 2\right\rfloor$ and $x_{k}^{n}=1$ for $k=A_{n}+\lfloor(n-$ $\left.\left.A_{n}\right) / 2\right\rfloor+1, \ldots, n$, these bounds follow by Lemma 4.1 and a routine application of the quadratic Chebyshev's inequality.

By the Markov property and the bounds (4.21), (4.22), to prove the statement it is enough to show that

$$
\begin{gathered}
\lim _{\ell \rightarrow+\infty} \lim _{n \rightarrow+\infty} \int d \mathcal{P}^{n} e^{i \sum_{h=1}^{r} \pi_{n}\left(X\left(t_{h}\right)\right)\left(\psi_{h}\right)} \mathbf{1}_{B_{\ell}^{1}}\left(\pi_{n}\left(X\left(t_{r}\right)\right)\right) \mathbf{1}_{B_{\varepsilon_{n}}^{2}}\left(X\left(t_{r}\right)\right) \\
\times \prod_{k=1}^{n} \mathbf{E}_{X_{k}^{n}\left(t_{r}\right)}^{\theta^{n}}\left[e^{i \psi_{r+1}\left(X\left(t_{r+1}-t_{r}\right)\right)}\right] \\
=\int \cdots \int \prod_{h=1}^{r+1} P_{t_{h}-t_{h-1}}\left(\eta_{h-1}, d \eta_{h}\right) e^{i \eta_{h}\left(\psi_{h}\right)}
\end{gathered}
$$

By remark 4.8 and again (4.21), (4.22) this follows from

$$
\begin{aligned}
\lim _{\ell \rightarrow+\infty} \lim _{n \rightarrow+\infty} \int d \mathcal{P}^{n} e^{i \sum_{h=1}^{r} \pi_{n}\left(X\left(t_{h}\right)\right)\left(\psi_{h}\right)} \\
\quad \times \mathbf{1}_{B_{\ell}^{1}}\left(\pi_{n}\left(X\left(t_{r}\right)\right)\right) \int P_{t_{r+1}-t_{r}}\left(\pi_{n}\left(X\left(t_{r}\right)\right), d \eta_{r+1}\right) e^{i \eta_{r+1}\left(\psi_{r+1}\right)} \\
=\lim _{\ell \rightarrow+\infty} \lim _{n \rightarrow+\infty} \mathbb{E}_{\omega}^{n}\left[e^{i \sum_{h=1}^{r} \omega\left(t_{h}\right)\left(\psi_{h}\right)}\right. \\
\left.\quad \times \mathbf{1}_{B_{\ell}^{1}}\left(\omega\left(t_{r}\right)\right) \int P_{t_{r+1}-t_{r}}\left(\omega\left(t_{r}\right), d \eta_{r+1}\right) e^{i \eta_{r+1}\left(\psi_{r+1}\right)}\right] \\
=\int \cdots \int \prod_{h=1}^{r+1} P_{t_{h}-t_{h-1}}\left(\eta_{h-1}, d \eta_{h}\right) e^{i \eta_{h}\left(\psi_{h}\right)} .
\end{aligned}
$$


Let $P_{t, \varepsilon}$ be the approximation of $P_{t}$ defined in (B.1). By Lemma B.1, (4.24) holds once we show

$$
\begin{aligned}
& \lim _{\varepsilon \rightarrow 0} \lim _{n \rightarrow+\infty} \mathbb{E}_{\omega}^{n}\left[e^{i \sum_{h=1}^{r} \omega\left(t_{h}\right)\left(\psi_{h}\right)} \int P_{t_{r+1}-t_{r}, \varepsilon}\left(\omega\left(t_{r}\right), d \eta_{r+1}\right) e^{i \eta_{r+1}\left(\psi_{r+1}\right)}\right] \\
& \quad=\int \cdots \int \prod_{h=1}^{r+1} P_{t_{h}-t_{h-1}}\left(\eta_{h-1}, d \eta_{h}\right) e^{i \eta_{h}\left(\psi_{h}\right)} .
\end{aligned}
$$

Since the map $\Omega \ni \omega \mapsto \int P_{t, \varepsilon}(\omega, d \eta) e^{i \eta(\psi)} \in \mathbf{C}$ is continuous, by the tightness of the marginal of $\left\{\mathbb{P}_{\omega}^{n}\right\}$ at the times $t_{1}, \ldots, t_{n}$ and the recursive assumption which identifies the cluster points of this law we can take the limit for $n \rightarrow+\infty$ above. Finally taking the limit $\varepsilon \rightarrow 0$ and using again Lemma B.1 we conclude the proof of (4.25).

\section{Appendix A. Topological complements}

For completeness, we discuss some details on the state space $\Omega$ both as topological and measurable space. Recalling that $\Omega$ has been endowed with the weakest topology such that the map $\omega \mapsto \omega(m \phi)$ is continuous for any $\phi \in C_{0}(0,1)$, a basis of this topology is the given by (see Megginson, 1998 Proposition 2.4.1) by the subsets of $\Omega$ of the form $\left\{\omega \in \Omega: \omega\left(m \phi_{1}\right) \in A_{1}, \ldots, \omega\left(m \phi_{n}\right) \in A_{n}\right\}$, where $n \in \mathbf{N}$, $\phi_{i} \in C_{0}(0,1)$, and $A_{i}$ are open subsets of $\mathbf{R}$. As follows by Proposition 2.4.8 of Megginson (1998) this topology is completely regular.

Lemma A.1. A set $K \subset \Omega$ is precompact if and only if $\sup _{\omega \in K} \omega(m)<+\infty$. Moreover, for each $\ell \in \mathbf{R}_{+}$the set $K_{\ell}:=\{\omega \in \Omega: \omega(m) \leq \ell\}$ is compact and the relative topology on $K_{\ell}$ is Polish.

Proof: We first show that for each $\ell \in \mathbf{R}_{+}$the set $K_{\ell}:=\{\omega \in \Omega: \omega(m) \leq \ell\}$ is compact and the relative topology on $K_{\ell}$ is Polish. Let $\Psi=\left\{\psi_{k}\right\}$ be a countable dense subset of $C_{0}(0,1)$ and set

$$
d(\omega, \eta):=\sum_{k} \frac{1}{2^{k}}\left(1 \wedge\left|\omega\left(m \psi_{k}\right)-\eta\left(m \psi_{k}\right)\right|\right) .
$$

We next prove that $d$ is a distance on $K_{\ell}$ inducing the relative topology. To this end, it is enough to show that given any open set $A \subset K_{\ell}$ and $\omega \in A$ there exists a $d$-ball centered in $\omega$ and contained in $A$. From the very definition of the topology, $A$ is of the form

$$
A=\bigcup\left\{\eta \in K_{\ell}: \eta\left(m \phi_{1}\right) \in U_{1}, \ldots, \eta\left(m \phi_{n}\right) \in U_{n}\right\}
$$

where $n \in \mathbf{N}, \phi_{i} \in C_{0}(0,1)$, and $U_{i}$ are open subsets of $\mathbf{R}$. If $\omega \in A$ then $\omega \in \bigcap_{i=1}^{n}\left\{\eta \in K_{\ell}: \eta\left(m \phi_{i}\right) \in U_{i}\right\}$ for some $n \in \mathbf{N}, \phi_{i}$, and $U_{i}$. Letting $\delta_{i}:=$ $\operatorname{dist}\left(\omega\left(m \phi_{i}\right), U_{i}^{c}\right)$ we now choose $\psi_{k(i)} \in \Psi$ such that $\left\|\psi_{k(i)}-\phi_{i}\right\|_{\infty}<\delta_{i} / \ell$ and $0<\rho<2^{-k(i)} \delta_{i} / \ell, i=1, \ldots, n$. Then $\omega \in\left\{\eta \in K_{\ell}: d(\eta, \omega)<\rho\right\} \subset\{\eta \in$ $\left.K_{\ell}: \eta\left(m \phi_{i}\right) \in U_{i}\right\}$.

To show that $K_{\ell}$ is separable it is enough to consider the collection of $\omega \in K_{\ell}$ which charges only rational points of $(0,1)$.

To prove compactness of $K_{\ell}$, we first observe that it is enough to show its sequencial compactness. Let $\left\{\omega_{n}\right\} \subset K_{\ell}$ be a sequence. By considering the restriction of $\omega_{n}$ to $[\delta, 1-\delta] \subset(0,1)$, using the compactness of Radon measures with uniformly bounded mass on $[\delta, 1-\delta]$, and a diagonal argument, we can find a Radon 
measure $\omega$ on $(0,1)$ and a subsequence (not-relabeled) $\left\{\omega_{n}\right\}$ vaguely convergent to $\omega$. Let $m_{k} \in C_{K}(0,1)$ be such that $m_{k} \uparrow m$. Then, by monotone convergence, $\omega(m)=\lim _{k} \omega\left(m_{k}\right)=\lim _{k} \lim _{n} \omega_{n}\left(m_{k}\right) \leq \ell$. Hence $\omega \in \Omega$. Finally by dominated convergence $\omega_{n}(m \phi) \rightarrow \omega(m \phi)$ for each $\phi \in C_{0}(0,1)$. Hence $\omega_{n} \rightarrow \omega$ in the topology of $K_{\ell}$.

To conclude the proof, we next show that if $K \subset \Omega$ is precompact then there exists $\ell \in \mathbf{R}_{+}$such that $K \subset K_{\ell}$. We argue by contradiction assuming that there exists a sequence $\left\{\omega_{n}\right\} \subset K$ such that $\omega_{n}(m) \rightarrow+\infty$; we will then construct a function $\phi \in C_{0}(0,1)$ such that $\omega_{n}(m \phi) \rightarrow+\infty$. By precompactness of $K$, we can assume $\omega_{n} \rightarrow \omega$ for some $\omega \in \Omega$. Let $\left\{x_{k}^{n}\right\}, k=1, \ldots,\left|\omega_{n}\right|$, be such that $\omega_{n}=\sum_{k} \delta_{x_{k}^{n}}$. Then either $\underline{\lim }_{n} \inf _{k} x_{k}^{n}=0$ or $\overline{\lim }_{n} \sup _{k} x_{k}^{n}=1$. We assume that the first alternative takes place and choose a subsequence of $\left\{x_{k}^{n}\right\}_{k}$ such that $\frac{1}{2} \geq x_{1}^{n} \geq x_{2}^{n} \geq \cdots$. We now set $\alpha_{n}:=\sum_{k} x_{k}^{n}$ and choose a subsequence such that $\alpha_{n} \uparrow+\infty$. It is not difficult to show that we can pick $h_{n} \rightarrow \infty$ such that $\sum_{k \leq h_{n}} x_{k}^{n} \geq \alpha_{n} / 2$ and $x_{h_{n}}^{n} \downarrow 0$. Finally, let $\phi \in C_{0}(0,1)$ be increasing on $(0,1 / 2]$ and such that $\phi\left(x_{h_{n}}^{n}\right)=\sqrt{1 / \alpha_{n}}$. Then, as $m(x) \geq x / 2$ for $x \in(0,1 / 2]$,

$$
\omega_{n}(m \phi) \geq \sum_{k} m\left(x_{k}^{n}\right) \phi\left(x_{k}^{n}\right) \geq \frac{1}{2} \sum_{k=1}^{h_{n}} x_{k}^{n} \phi\left(x_{k}^{n}\right) \geq \frac{1}{2} \phi\left(x_{h_{n}}^{n}\right) \sum_{k=1}^{h_{n}} x_{k}^{n} \geq \frac{1}{4} \sqrt{\alpha_{n}}
$$

which completes the proof.

Lemma A.2. Let $\mathcal{C}$ be the family of subsets of $\Omega$ of the form

$$
C=\left\{\omega \in \Omega: \omega\left(U_{1}\right)=n_{1}, \ldots, \omega\left(U_{k}\right)=n_{k}\right\},
$$

for some $k \in \mathbf{N}, n_{i} \in \mathbf{Z}_{+}$, and $U_{i}$ open subset of $(0,1)$ such that $U_{i} \subset \subset(0,1)$. Then $\mathcal{C}$ is $\pi$-system that generates the Borel $\sigma$-algebra $\mathcal{B}(\Omega)$.

Proof: The family $\mathcal{C}$ is obviously closed for finite intersections. To show that $\sigma(\mathcal{C}) \subset$ $\mathcal{B}(\Omega)$ it is enough to show that for each $U \subset \subset(0,1)$ open and $n \in \mathbf{N}$ the set $\{\omega \in \Omega: \omega(U)=n\}$ is Borel subset of $\Omega$. Let $\phi_{k} \in C_{K}(0,1)$ such that $\phi_{k} \uparrow \mathbf{1}_{U}$. Then

$$
\{\omega \in \Omega: \omega(U)=n\}=\bigcap_{m} \bigcup_{k} \bigcap_{h \geq k}\left\{\omega \in \Omega: \omega\left(\phi_{h}\right) \in\left(n-\frac{1}{m}, n+\frac{1}{m}\right)\right\} \in \mathcal{B}(\Omega) .
$$

To prove the inclusion $\sigma(\mathcal{C}) \supset \mathcal{B}(\Omega)$, let us first prove that, given $\ell \in \mathbf{N}$, the set $K_{\ell}:=\{\omega \in \Omega: \omega(m) \leq \ell\}$ belongs to $\sigma(\mathcal{C})$. To this end, we say that a function $\phi:(0,1) \rightarrow \mathbf{R}$ is simple if it has the form $\phi=\sum_{i=1}^{n-1} \alpha_{i} \mathbf{1}_{\left[x_{i}, x_{i+1}\right)}$ for some $n \in \mathbf{N}, \alpha_{i} \in \mathbf{R}$, and $0<x_{1}<\cdots<x_{n}<1$. Then it straightforward to show that, for each simple function $\phi$, the map $\omega \mapsto \omega(\phi)$ is $\sigma(\mathcal{C})$-measurable. Pick now a sequence $m_{k}$ of simple functions such that $m_{k} \uparrow m$. Then, by monotone convergence, $K_{\ell}=\bigcap_{k}\left\{\omega \in \Omega: \omega\left(m_{k}\right) \leq \ell\right\} \in \sigma(\mathcal{C})$.

By observing that an open set $A \subset \Omega$ can be written as $A=\bigcup_{\ell \in \mathbf{N}}\left(A \cap K_{\ell}\right)$ and using Lemma A.1, to conclude the proof it is enough to show that the distance $d$ in (A.1) is $\sigma(\mathcal{C}) \times \sigma(\mathcal{C})$ measurable. To this end given $\psi \in \Psi$ and $U \in \mathcal{B}(\mathbf{R})$ we show that the set $\left\{\omega \in K_{\ell}: \omega(m \psi) \in U\right\}$ belongs to $\sigma(\mathcal{C})$. Pick a sequence $\phi_{k}$ of simple functions such that $\phi_{k} / m \rightarrow \psi$ uniformly in $(0,1)$. In particular $\omega\left(\phi_{k}\right) \rightarrow \omega(m \psi)$ uniformly for $\omega \in K_{\ell}$. Then

$$
\left\{\omega \in K_{\ell}: \omega(m \psi) \in U\right\}=\bigcup_{k} \bigcap_{h \geq k}\left\{\omega \in K_{\ell}: \omega\left(\phi_{h}\right) \in U\right\} \in \sigma(\mathcal{C}) .
$$


Lemma A.3. Let $P_{1}, P_{2}$ be two probabilities on $(\Omega, \mathcal{B}(\Omega))$ such that

$$
\int P_{1}(d \omega) e^{i \omega(\psi)}=\int P_{2}(d \omega) e^{i \omega(\psi)}, \quad \psi \in C_{K}(0,1) .
$$

Then $P_{1}=P_{2}$.

Proof: In view of Lemma A.2, the proof is achieved by the argument in Theorem $\S 29.14$ of Fristedt and Gray (1997).

Lemma A.4. For each $t \geq 0$ and $\omega \in \Omega$ there exists a unique probability $P_{t}^{0}(\omega, \cdot)$ on $(\Omega, \mathcal{B}(\Omega))$ such that

$$
\int P_{t}^{0}(\omega, d \eta) e^{i \eta(\psi)}=\prod_{x \in \omega} \int p_{t}^{0}(x, d y) e^{i \psi(y)}, \quad \psi \in C_{K}(0,1) .
$$

Proof: As $P_{0}^{0}(\omega, \cdot)=\delta_{\omega}$, we consider $t>0$. Let $\left(x_{k}\right)_{k=1, \ldots,|\omega|} \in(0,1)^{|\omega|}$ be such that $\omega=\sum_{k} \delta_{x_{k}}$. Let $Q$ be the product measure on $\Xi:=\prod_{k}[0,1]$ with marginals $p_{t}^{0}\left(x_{k}, \cdot\right)$. Elements of $\Xi$ are denoted by $y=\left(y_{k}\right)$. Let us first prove that

$$
Q(F)=1, \quad F:=\left\{y \in \Xi: y_{k} \in(0,1) \text { for finitely many } k\right\}
$$

Indeed,

$$
\begin{gathered}
\sum_{k} Q\left(y_{k} \in(0,1)\right)=\sum_{k} \mathbf{P}_{x_{k}}^{0}(X(t) \in(0,1))=\sum_{k} \mathbf{P}_{x_{k}}^{0}(\tau>t) \\
\leq \frac{1}{t} \sum_{k} \mathbf{E}_{x_{k}}^{0}(\tau)=\frac{1}{t} \sum_{k} m\left(x_{k}\right)=\frac{\omega(m)}{t}<+\infty
\end{gathered}
$$

and (A.3) follows by Borel-Cantelli lemma.

We now define the map $\pi: \Xi \rightarrow \Omega$ by $\pi(y)=\sum_{k} \delta_{y_{k}}(\cdot \cap(0,1))$ if $y \in F$ and $\pi(y)=0$ if $y \notin F$. Observe that $\pi$ is $\mathcal{B}(\Omega)-\mathcal{B}(\Xi)$ measurable since $F \in \mathcal{B}(\Xi)$ and the map $F \ni y \mapsto \pi(y)$ is a pointwise limit of continuos maps.

By setting $P_{t}^{0}(\omega, \cdot):=Q \circ \pi^{-1}$, it satisfies (A.2) in view of (A.3) and the change of variable formula. Uniqueness follows by Lemma A.3.

Given $T>0$ we let $\Omega_{T}:=C([0, T] ; \Omega)$ be the set of the $\Omega$ valued continuous functions. We consider $\Omega_{T}$ endowed with the compact-open (uniform) topology and the corresponding Borel $\sigma$-algebra $\mathcal{B}\left(\Omega_{T}\right)$. Let $\mathcal{C}_{T}$ be the family of subsets of $\Omega_{T}$ of the form

$$
C=\left\{\omega \in \Omega_{T}: \omega\left(t_{1}\right) \in B_{1}, \ldots, \omega\left(t_{k}\right) \in B_{k}\right\},
$$

for some $k \in \mathbf{N}, 0 \leq t_{1}<\cdots<t_{k} \leq T$, and $B_{i} \in \mathcal{B}(\Omega), i=1, \ldots, k$.

Lemma A.5. The family $\mathcal{C}_{T}$ is $\pi$-system that generates the Borel $\sigma$-algebra $\mathcal{B}\left(\Omega_{T}\right)$. In particular, a probability on $\left(\Omega_{T}, \mathcal{B}\left(\Omega_{T}\right)\right.$ is uniquely characterized by its finite dimensional distributions.

Proof: Since $\Omega$ is a completely regular topological spaces with metrizable compacts, the lemma follows from Corollary 2.6 of Jakubowski (1986) applied in the present context of $C([0, T] ; \Omega)$ instead of $D([0, T] ; \Omega)$. Note indeed that condition $(2.13)$ in Jakubowski (1986) follows from Lemma A.1. 


\section{Appendix B. Approximation of the semigroup}

We here discuss the approximation of the semigroup $P_{t}$ used in Section 4.4. Given $\varepsilon>0$ let $\chi_{\varepsilon} \in C_{K}(0,1)$ such that $0 \leq \chi_{\varepsilon} \leq 1, \chi_{\varepsilon}(x)=1$, for $x \in(\varepsilon, 1-\varepsilon)$. For $\omega \in \Omega$ we denote by $\omega_{\varepsilon}$ the thinning of $\omega$ obtained by erasing independently each particle $x \in \omega$ with probability $1-\chi_{\varepsilon}(x)$. In particular, $\left|\omega_{\varepsilon}\right|<+\infty$ and $\operatorname{supp} \omega_{\varepsilon} \subset \operatorname{supp} \chi_{\varepsilon}$. For $\omega \in \Omega$ and $t \in \mathbf{R}_{+}$we define the probability $P_{t, \varepsilon}(\omega, \cdot)$ on $\Omega$, as the mixture of $P_{t}(\eta, \cdot)$ with $\eta \in \Omega$ sampled according to the law of the thinning $\omega_{\varepsilon}$, that is

$$
P_{t, \varepsilon}(\omega, B):=\iint_{\eta_{1}+\eta_{2} \in B} \Pi_{\mu_{t}^{\lambda}}\left(d \eta_{1}\right) P_{t, \varepsilon}^{0}\left(\omega, d \eta_{2}\right), \quad B \in \mathcal{B}(\Omega),
$$

where $P_{t, \varepsilon}^{0}(\omega, \cdot)$ is the probability on $\Omega$ characterized by,

$$
\int P_{t, \varepsilon}^{0}(\omega, d \eta) e^{i \eta(\psi)}=\prod_{x \in \omega}\left(\chi_{\varepsilon}(x) \int p_{t}^{0}(x, d y) e^{i \psi(y)}+1-\chi_{\varepsilon}(x)\right), \psi \in C_{K}(0,1) .
$$

Lemma B.1. For each $\varepsilon>0, t>0$, and each $f \in C(\Omega)$ bounded, the map $\omega \mapsto P_{t, \varepsilon}(\omega, f)$ is continuous. Furthermore $P_{t, \varepsilon}(\omega, f)$ converges as $\varepsilon \rightarrow 0$ to $P_{t}(\omega, f)$ pointwise in $\omega$ and uniformly for $|\omega| \leq \ell, \ell \in \mathbf{N}$.

Proof: In view of (B.1) and dominated convergence it is enough to prove the statement for the map $\omega \mapsto P_{t, \varepsilon}^{0}(\omega, f)$. Since $p_{t}^{0}$ is a Feller transition function, the continuity of $\omega \mapsto P_{t, \varepsilon}^{0}(\omega, f)$ readily follows from the definition. To prove the pointwise convergence $P_{t, \varepsilon}^{0}(\omega, f) \rightarrow P_{t}^{0}(\omega, f)$, we observe that, as follows from (A.3),

$$
\lim _{\ell \rightarrow+\infty} \varlimsup_{\varepsilon \rightarrow 0} P_{t, \varepsilon}^{0}(\omega,\{\eta:|\eta|>\ell\}) \leq \lim _{\ell \rightarrow+\infty} P_{t}^{0}(\omega,\{\eta:|\eta|>\ell\})=0,
$$

which implies the tightness of the family $\left\{P_{t, \varepsilon}^{0}(\omega, \cdot)\right\}_{\varepsilon>0}$. Moreover, by a direct computation, for each $\psi \in C_{K}(0,1)$

$$
\lim _{\varepsilon \rightarrow 0} \int P_{t, \varepsilon}^{0}(\omega, d \eta) e^{i \eta(\psi)}=\prod_{x \in \omega} \int p_{t}^{0}(x, d y) e^{i \psi(y)}=\int P_{t}^{0}(\omega, d \eta) e^{i \eta(\psi)} .
$$

By Prokhorov's theorem (see e.g. Smoljanov and Fomīn, $1976 \S 5$, Thm. 2 for the present setting of a completely regular topological space with metrizable compacts) and Lemma A.3 we then conclude.

Finally, the uniform convergence $P_{t, \varepsilon}^{0}(\omega, f) \rightarrow P_{t}^{0}(\omega, f)$ on $|\omega| \leq \ell$ follows from the convergence of $\chi_{\varepsilon} \rightarrow \mathbf{1}_{(0,1)}$ uniform on compact subsets of $(0,1)$.

\section{Acknowledgements}

This work was motivated by G. Ciccotti who asked how to construct boundary driven models on the continuum. It is our duty and pleasure to thank A. Teixeira who explained us the graphical construction presented in Section 3.2.

\section{References}

S. Albeverio, Yu. G. Kondratiev and M. Röckner. Analysis and geometry on configuration spaces. J. Funct. Anal. 154 (2), 444-500 (1998). MR1612725.

L. Bertini, A. De Sole, D. Gabrielli, G. Jona-Lasinio and C. Landim. Macroscopic fluctuation theory. Rev. Mod. Phys. 87, 593-636 (2015). DOI: 10.1103/RevModPhys.87.593. 
P. Billingsley. Convergence of probability measures. Wiley Series in Probability and Statistics: Probability and Statistics. John Wiley \& Sons, Inc., New York, second edition (1999). ISBN 0-471-19745-9. MR1700749.

A. N. Borodin and P. Salminen. Handbook of Brownian motion-facts and formulae. Probability and its Applications. Birkhäuser Verlag, Basel, second edition (2002). ISBN 3-7643-6705-9. MR1912205.

R. L. Dobrušin. On Poisson's law for distribution of particles in space. Ukrain. Mat. Ž. 8, 127-134 (1956). MR0081016.

P. A. Ferrari and L. R. G. Fontes. The net output process of a system with infinitely many queues. Ann. Appl. Probab. 4 (4), 1129-1144 (1994). MR1304777.

M. Fradon, S. Roelly and H. Tanemura. An infinite system of Brownian balls with infinite range interaction. Stochastic Process. Appl. 90 (1), 43-66 (2000). MR1787124.

B. Fristedt and L. Gray. A modern approach to probability theory. Probability and its Applications. Birkhäuser Boston, Inc., Boston, MA (1997). ISBN 0-81763807-5. MR1422917.

S. Hiraba. Infinite Markov particle systems with singular immigration; martingale problems and limit theorems. Osaka J. Math. 33 (1), 145-187 (1996). MR1381624.

A. Jakubowski. On the Skorokhod topology. Ann. Inst. H. Poincaré Probab. Statist. 22 (3), 263-285 (1986). MR871083.

L. Kruk, J. Lehoczky, K. Ramanan and S. Shreve. An explicit formula for the Skorokhod map on [0,a]. Ann. Probab. 35 (5), 1740-1768 (2007). MR2349573.

K. Mallick. The exclusion process: A paradigm for non-equilibrium behaviour. Physica A: Statistical Mechanics and its Applications 418, 17-48 (2015). DOI: 10.1016/j.physa.2014.07.046.

R. E. Megginson. An introduction to Banach space theory, volume 183 of Graduate Texts in Mathematics. Springer-Verlag, New York (1998). ISBN 0-387-98431-3. MR1650235.

D. Revuz and M. Yor. Continuous martingales and Brownian motion, volume 293 of Grundlehren der Mathematischen Wissenschaften [Fundamental Principles of Mathematical Sciences]. Springer-Verlag, Berlin (1991). ISBN 3-540-52167-4. MR1083357.

T. Shiga and Y. Takahashi. Ergodic properties of the equilibrium process associated with infinitely many Markovian particles. Publ. Res. Inst. Math. Sci. 9, 505-516 (1973/74). MR0402988.

O. G. Smoljanov and S. V. Fomīn. Measures on topological linear spaces. Usephi Mat. Nauk 31 (4, (190)), 3-56 (1976). MR0420764.

H. Tanemura. A system of infinitely many mutually reflecting Brownian balls in $\mathbf{R}^{d}$. Probab. Theory Related Fields 104 (3), 399-426 (1996). MR1376344.

S. R. S. Varadhan. Stochastic processes, volume 16 of Courant Lecture Notes in Mathematics. Courant Institute of Mathematical Sciences, New York; American Mathematical Society, Providence, RI (2007). ISBN 978-0-8218-4085-6. MR2354349. 